\title{
X-Ray Microcomputed Tomography in Additive Manufacturing: A Review of the Current Technology and Applications
}

\author{
Anton du Plessis, ${ }^{1,2}$ Igor Yadroitsev, ${ }^{3}$ Ina Yadroitsava, and Stephan G. Le Roux ${ }^{1}$
}

\begin{abstract}
X-ray microcomputed tomography (microCT) has become an established method of testing and analyzing additively manufactured parts in recent years, being especially useful and accurate for dimensional measurement and porosity analysis. While this nondestructive analysis method is gaining traction among additive manufacturing (AM) researchers and engineers, the capabilities of the method are not yet fully appreciated and are still being developed. This review aims to summarize the many diverse ways this technique has been applied to AM, including new and specialized applications. Examples are shown of many of these newly developed methods, while also discussing the practicality and limitations of each. The review ends with perspectives on the most time- and cost-effective ways to make use of microCT for various AM applications from R\&D up to industrial production, with suggestions for scan strategies for different types of analyses.
\end{abstract}

Keywords: microCT, X-ray tomography, nondestructive testing, additive manufacturing, powder bed fusion

\section{Introduction}

AdDITIVE MANUFACTURING (AM) is a layer-by-layer manufacturing method that has grown considerably in recent years, especially for producing functional metal parts for critical applications in medical and aerospace industries. ${ }^{1,2}$ Powder bed fusion $(\mathrm{PBF})$ is the term used to describe specifically metal AM using a laser (LPBF) or electron beam (EB-PBF) to melt tracks and layers for the manufacture of complex shaped parts. ${ }^{3}$

Despite the huge progress in recent years in this technology, and its increasing adoption, there remain some production issues. These issues include unwanted porosity from incorrect processing parameters or build conditions, surface roughness or other surface imperfections, deformation caused by residual stresses, and mechanical properties that are anisotropic, for example. These imperfections can be exaggerated due to the complex nature of the designs possible by PBF. Due to these challenges, process qualification is required and manufactured parts require careful testing, especially for high value and critical parts such as those for aerospace or medical applications.
X-ray microcomputed tomography (microCT) is becoming an established technique for nondestructive analysis in various fields of application. In materials sciences, its increasingly widespread use was reviewed in Maire and Withers, ${ }^{4}$ which makes it clear that the method has evolved from a qualitative imaging technique in the past to a mature and quantitative analytical technique in recent years. It finds particular use as a high-quality and nondestructive analysis tool in various industrial applications as reviewed in De Chiffre et al. ${ }^{5}$ In this review of industrial applications, a section was devoted to AM, demonstrating quality control of complex parts and latticed parts-stating that this is the only method suitable to nondestructively analyze additively manufactured parts with internal cavities and porosities.

Since PBF allows the manufacturing of objects with complex shapes and internal design, including lattice structures and foams, microCT is very useful to quantify the porosity, to study the cell morphology, and to evaluate internal and external surface roughness, overall structural integrity, and the extent and distribution of internal defects. 6,7

\footnotetext{
${ }^{1}$ CT Scanner Facility, Stellenbosch University, Stellenbosch, South Africa.

${ }^{2}$ Physics Department, Stellenbosch University, Stellenbosch, South Africa.

${ }^{3}$ Department of Mechanical and Mechatronic Engineering, Central University of Technology, Bloemfontein, South Africa.

(c)Anton du Plessis et al. 2018; Published by Mary Ann Liebert, Inc. This Open Access article is distributed under the terms of the Creative Commons License (http://creativecommons.org/licenses/by/4.0), which permits unrestricted use, distribution, and reproduction in any medium, provided the original work is properly cited.
} 
While the use of X-ray microCT is gaining acceptance in the AM community, it is used most often to measure porosity and confirm dimensional measurements, as discussed in Thompson et al. ${ }^{8}$ This review article on the use of X-ray computed tomography in AM documented the historical development of the two technologies and their combined use, while focusing mainly on porosity inspection and dimensional measurement for quality controls. It was concluded that the main drawbacks to the wider uptake of the technique are costs and lack of standards.

The scope of the present review article is to demonstrate and discuss all the varied ways microCT has been used in AM, in addition to the abovementioned porosity and dimensional measurements. The aim of this review is therefore to broaden the general understanding of how this technique can be used to complement and support AM, which is not generally known and which is still under continuous development. This includes various interesting applications and new developments applicable to AM at different levels, from powder characterization to surface roughness assessment and to image-based simulations.

This summary of the capabilities will hopefully provide insight into how best to make use of this powerful technique, allowing a proper selection of microCT testing strategy for a particular application. Based on the various applications demonstrated and discussed in the review, suggestions are made of microCT testing strategies for most cost-effective use of the technique. Considering the fast progress in both fields of X-ray
microCT and in AM, regular reviews of the synergies between these two technologies will continue to remain important in the next few years as advances are made in both. While the discussions are broadly applicable to all AM, the focus of the work is on the most critical applications for aerospace and medical applications, that is, metal PBF, in particular LPBF.

\section{Background}

\section{Basic principles of $X$-ray microCT}

$\mathrm{X}$-ray microCT works on the principle of irradiating a sample with a beam of X-rays, measuring the subsequent absorption X-ray image, and repeatedly acquiring such images as the sample rotates. The X-ray absorption (so-called projection) images represent views of the sample from many angles, providing internal detail due to the penetration of X-rays. ${ }^{9}$ The acquired images are then used in a mathematical reconstruction process to generate a volumetric data set. This volume comprises voxels (volumetric pixels) with the brightness of each pixel related to the X-ray density of the material it represents (X-ray density depends on physical density and atomic mass).

A schematic of the process is shown in Figure 1, which is a modified version taken from a tutorial review of X-ray CT in food sciences, ${ }^{10}$ which also describes the fundamentals of the process in more detail. The schematic shown in Figure 1 is a representation of most typical laboratory microCT setups, with a microfocus X-ray source, a rotating sample stage, a planar
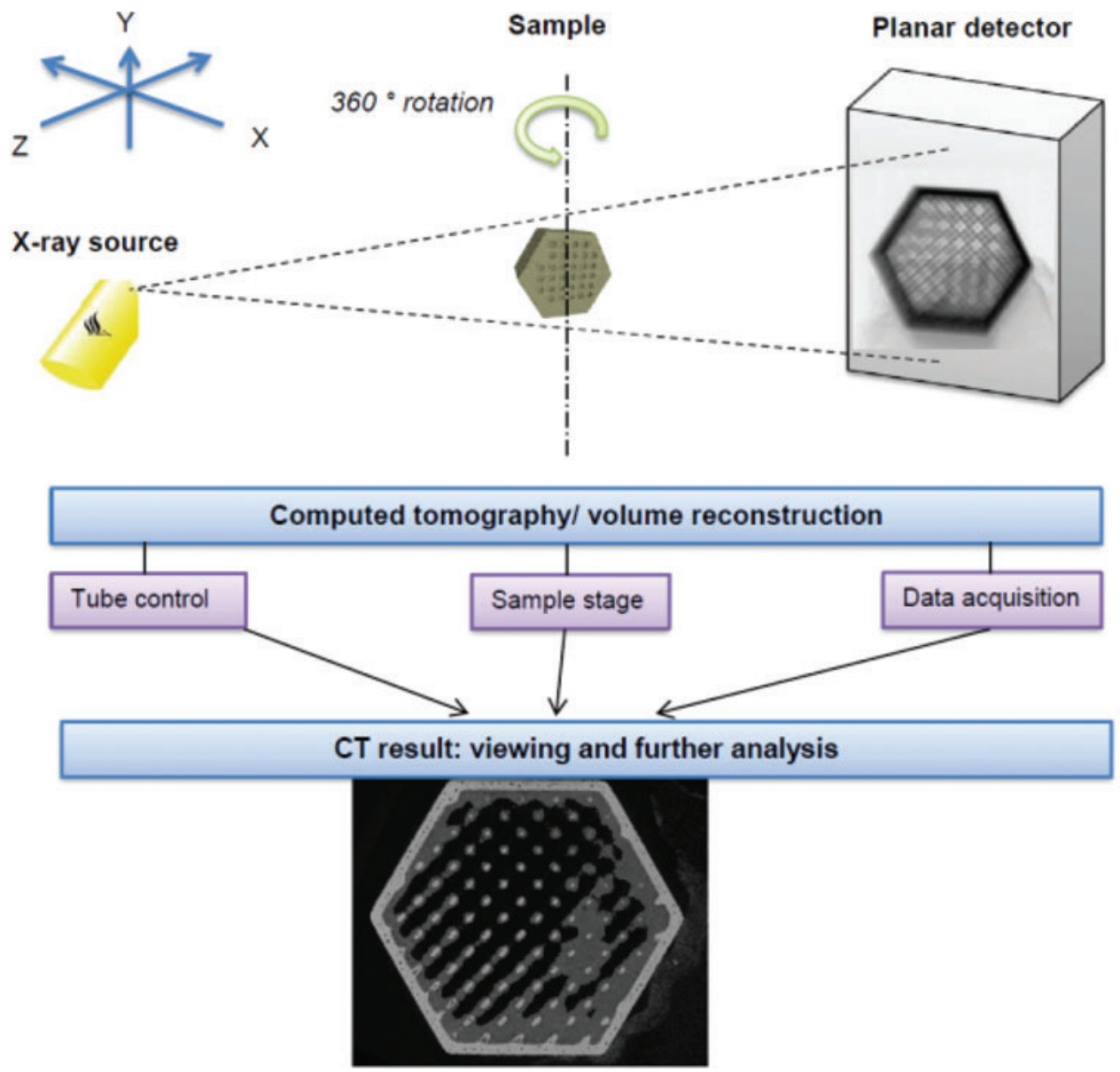

FIG. 1. Schematic of an X-ray microCT scan. microCT, microcomputed tomography. Color images available online at www.liebertpub.com/3dp 
detector, and integrated software, to acquire images and reconstruct the volume data. After scanning and reconstruction, data visualization and analysis are further performed, typically in dedicated software. In this schematic, a polyamide (polymer) hexagonal sample with internal lattice structure is shown, which was built by selective laser sintering. The CT slice image shows the presence of remaining powder, indicating a simple yet powerful visualization by CT, without any postprocessing.

A series of guidelines for producing good-quality scans is provided in du Plessis et al. ${ }^{11}$ These guidelines are meant for biological samples but are also valid more generally, and are therefore also applicable to additive manufactured parts. For metal AM parts, high beam voltages and beam filtering are also important factors to consider and some guidelines are also presented in Ref. ${ }^{12}$

\section{Current technology: types of systems-hardware and software}

A typical laboratory microCT setup, as described in du Plessis et al., ${ }^{13}$ allows the acquisition of microCT scans with resolution down to $5 \mu \mathrm{m}$, and submicron CT or nanoCT down to $0.5 \mu \mathrm{m}$. Some variations of systems exist with regard to the types of detectors (bit depth, pixel size, sensitivity), X-ray sources (maximum voltage, brightness, stability, smallest spot size), and translation and rotation hardware (stability, accuracy). These variations in practice extend from small desktop microCT systems with limited sample size capabilities to large room-sized cabinets.

One of the latest developments is helical scanning as demonstrated in Seifi et al., ${ }^{14}$ which effectively removes some types of image artifacts and allows acquisition of single volumes of elongated samples, instead of stitching multiple scans. Another new development, which can be very useful in practice, is offaxis CT: rotation around an arbitrary selected point in the sample. This allows higher resolution scans to be obtained with less sample mounting limitations.

Besides the typical systems using geometric magnification, some systems utilize a different concept where a collimated beam is used, allowing for higher resolution imaging of small regions inside an object. These are excellent for high-resolution research studies, but not for quality inspections due to long scan times. Similarly, synchrotron tomography is extremely useful for research studies, due to the high-resolution, high X-ray flux and fast scan times possible, see for example, Refs. ${ }^{15,16}$ However, beam time is limited and not easily or fast accessible at large-scale facilities.

Reconstruction of acquired 2D images into 3D volumetric data is typically performed using system-supplied software, based on variations of a filtered back-projection algorithm. ${ }^{17}$ Much work has recently been performed in new reconstruction methods, including iterative reconstruction, which holds promise for improved data quality in future and is an ongoing development, see for example, Biguri et al. ${ }^{18}$

Visualization and analysis are typically quite computationally challenging, hence the need for dedicated software packages and associated high-end computing facilities. The most widely used software packages for voxel data analysis are Volume Graphics VGStudio Max and FEI Avizo. Due to the cost of typical hardware and the technical experience and skill required to obtain good scans, generate good reconstructions, and allow access to suitable computing power and software, multiuser facilities such as in du Plessis et al. ${ }^{13}$ are often used cost effectively and should be used more often by AM engineers. This is one way to lower the cost barrier to the use of the technology.

\section{Applications}

\section{Porosity and defect analysis}

Despite the advantages of AM, various forms of defects can occur, which can be detrimental to the mechanical properties of the produced parts. Pores can be caused by many different physical processes and can often be due to different melt pool dynamics that depend on process parameters and conditions as discussed in Khairallah et al. ${ }^{19}$ Complex shapes and internal structures of PBF parts also result in worse inspectability of the defects. ${ }^{20}$

Many of the defects can be attributed to nonoptimal build conditions and powder properties. Since LPBF is a process involving selective laser fusion of a predeposited powder layer, it can be expected that the quality of the powder layer is crucial. Therefore, the powder particle shapes, the presence of satellites, the particle size distribution, oxidation level, humidity, static charge, and so on, which all can influence the flowability of the powder, packing density, and homogeneity of the deposited powder layer, ultimately impact on the process and resulting porosity. ${ }^{21-24}$

Parameter optimization is required for each powder material and system used as demonstrated, for example, in Refs. ${ }^{3,25-27}$ Recent studies investigated the porosity in AM parts over a range of process conditions demonstrating keyhole mode and lack of fusion porosity. ${ }^{28-30}$ Keyhole mode porosity represents a trail of voids in the wake of the laser beam and occurs when laser energy input is too high, resulting in evaporation of the metal and deep penetration; the collapse of the vapor cavity leads to pore formation. ${ }^{31}$ Keyhole pores have irregular shape and relatively large size-more than $100 \mu \mathrm{m} .^{32}$ This was visualized by microCT for a range of process parameters in laser welding. ${ }^{33}$ In another study, keyhole mode porosity was analyzed by sectioning and microCT. ${ }^{31}$

Lack of fusion porosity is usually irregular shaped and can contain internal unmelted or partially sintered powder particles. ${ }^{34,35}$ Lack-of-fusion porosity is a result of insufficient overlap of successive melt pools between layers or nonoptimal hatch distance between the tracks. ${ }^{21,36}$ Denudation of the substrate $^{19,21}$ can lead to nonuniform geometrical characteristics of consecutive tracks-thus gaps between tracks can exist at certain hatch distances. As a result, pores elongated in shape along the $X Y$-plane were found in sintered LPBF layers and 3D samples. ${ }^{37-39}$

Incomplete part consolidation also can be due to poor wetting and porosity along the edges of melt pools as was found in Tang et al. ${ }^{36} \mathrm{~A}$ different type of defect resembling chains of pores in building $Z$-direction were found by $\mathrm{X}$-ray tomography ${ }^{22}$ in LPBF samples produced at a high scan speed, which could be associated with the balling effect. Laser beam energy was insufficient to melt material in a high layer thickness of several consecutive layers, and inhomogeneity in various regions leads to different melting and solidifying behavior and ultimately to irregular porosity. ${ }^{32}$

At low-energy input, a shallow molten pool is formed and irregular pores with large size were found in LPBF samples. ${ }^{40}$ When thick layers were processed, spherical and irregular pores were also found. ${ }^{36,41} \mathrm{~A}$ wide range of process parameters 
with high levels of porosity and good images of lack of fusion porosity containing trapped unmelted particles are shown in Refs. $22,30,35,42$

Porosity shape dependence on scanning speed at fixed laser power was investigated in Panwisawas et $a l^{43}$ It was found that there is a clear threshold scanning speed difference between where intralayer pores with spherical, elliptical, or triangular morphologies exist and where interlayer pores with horizontal elongated form. It was shown that thermal fluid dynamics has a great impact on the temperature fields and melt interface on the evolution of porosity in LPBF material.

The procedure for measurement and characterization of porosity in LPBF small parts using X-ray CT scans may depend on image analysis procedures and one methodology is described in Cai et al. ${ }^{44}$ It was mentioned that the reconstruction of internal structure by CT scans enhances the understanding of the process parameters on LPBF porosity and gives the possibility to analyze spatial distribution of the pores, its shapes and sizes and to optimize process parameters on that basis.

Metallurgical pores, or small spherical pores less than $100 \mu \mathrm{m}$ in LPBF samples, were found at low scanning speed and high laser power, that is, high linear energy input. ${ }^{32,40} \mathrm{It}$ was indicated in Panwisawas et al. $^{43}$ that small amounts of trapped gas between powder particles act as a nucleation site for spherical pores. Gas entrapped in the melt pool originating from the feedstock powder was identified in recent studies, such as in Cunningham et al. ${ }^{45}$ where the presence of porosity in the powder was correlated with porosity in the built EB-PBF parts.

For another study in LPBF, pores in the feed stock powder were observed and mentioned that they can cause porosity in the final part. ${ }^{46}$ Rapid melting/cooling during LPBF results in such trapped gas pores having no time to outgas and therefore remaining in the part. The authors also mentioned the disadvantage of small pores less than $8 \mu \mathrm{m}$, which cannot be detected by typical microCT scans-for such small pores, it was determined that cross-sectioning and optical microscopy seem to be more reliable in this case. ${ }^{46}$

A high laser power leads to a high temperature, which, in turn, contributes to strong flows and spattering of the molten pool. ${ }^{19,47}$ Irregular sintered layers and satellites can influence the homogeneity of the next deposited powder layer and this can be a cause of pore formation. During LPBF, the laser scans a thin predeposited powder layer. As shown in Refs., ${ }^{35,41,43}$ processing of thick powder layers can cause inhomogeneity in powder packing, which can cause pores in 3D LPBF objects. It was found that when the deposited powder was agglomerated, the inhomogeneous powder layer led to high porosity in sintered samples. Processing homogenous powder layers at the same process parameters resulted in fully dense samples as shown in Kouprianoff et al. ${ }^{48}$

Scanning and building strategies also influence the porosity of the samples, as was shown with microCT investigations of LPBF parts. ${ }^{49-51}$ A contouring scan strategy can be a reason of pores between the core part and the contouring area. An increased porosity in bottom areas of the samples built with support structures was found in an article by Damon et al. ${ }^{50}$

Stop/start cycles during the build of the part can cause planar porosity as was shown in du Plessis et al. ${ }^{52}$ In this work, the thin layered defect in the plane of the build platform could be detected and quantified by microCT in a complex part, before and after hot isostatic pressing (HIP). This showed that much of the planar porosity remains after the
HIP process. This is therefore one very important type of porosity to identify nondestructively and to eliminate from the process if possible. HIP is often used to remove gas porosity, even closing pores as large as $4 \mathrm{~mm}$ to less than $10 \mu \mathrm{m}$ as shown for casting porosity, ${ }^{53}$ but when pores are connected to the surface, the method is ineffective.

At optimal conditions, LPBF samples have fairly randomly distributed defects such as small spherical gas pores and defects in the form of microshrinkages between the connected layers, and the mechanical behavior of the samples is not determined by the presence of these small defects, but mostly by specific microstructure after LPBF. ${ }^{54,55}$

A recent excellent example of the use of X-ray microCT to visualize and quantify porosity and build defects in small EBPBF samples is provided in Tammas-Williams et al. ${ }^{56}$ These authors demonstrate how the amount of porosity and its 3D distribution can be related to the process parameters, and they mention how this information can be used to provide feedback and optimize the process parameters. Most work thus far by manufacturers of AM systems has optimized processes based only on microstructure. This study was for electron beam melting and typical porosity was in the region of $0.2 \%$.

A similar type of study was reported in Maskery et al. ${ }^{57}$ for LPBF parts, with typical porosity of $0.1 \%$. In this study, various heat treatments were used on the same samples, which affected the microstructure but left pores unaffected. In a similar study, AM samples were analyzed by microCT before and after HIP and subsequently after heat treatment cycles. Interestingly, the pores disappear below the resolution limit of the microCT scan after HIP, but regrow to detectable sizes after heat treatment. While the amount of regrowth is limited, it does indicate the presence of porosity even after HIPping, despite being small.

Clearly, microCT can be used to identify porosity in small AM samples, but how does this relate to real parts? The larger part size of typical functional components limits the best possible resolution in microCT, and hence, small pores can be missed. The major question is: which small pores are missed in any particular case? In an ideal scenario, where a perfect quality scan is analyzed, the typical minimum pore size that is positively identified is at least 3 voxels wide, that is, in 3D it comprises at least 27 voxels. Taking this "general rule" as minimum detectable pore size, a scan at $10 \mu \mathrm{m}$ voxel size will quantify all pores $>30 \mu \mathrm{m}$ in diameter.

The best voxel resolution depends on the part size by another "general rule" for typical geometrical magnification microCT systems: the best possible resolution is 2000 times smaller than the widest part of the sample. This means a $20 \mathrm{~mm}$ part has the best possible voxel size of $10 \mu \mathrm{m}$. This rule is valid for whole parts and sometimes varies depending on the angle of scanning required to eliminate artifacts.

In addition to this size/resolution/detail detectability problem, larger parts suffer from X-ray penetration problems (depending on parameters used), which can result in image quality issues and hence decreased data quality. Despite these drawbacks, the method can be used effectively for quality control purposes as demonstrated in Refs. ${ }^{51,58}$

In one of these cases, a reference porosity sample was used and it was demonstrated that a further higher resolution analysis of the microporosity is possible by sectioning the part, as the part size limits the resolution obtained by microCT. This methodology can be used as part of a strategy to check the 3D 
distribution of porosity and this information can be used to minimize porosity by varying process parameters. In this case, most of the detected microporosity was located on the underside of the part and is related to the surface connected to support structures similar to those mentioned in Ref. ${ }^{50}$ One strategy to reduce this is to change the process parameters at these locations, or to add material in the design, and use postprocess machining to remove this near-surface porous material.

The most useful and most widely used application of microCT in $\mathrm{AM}$ is the nondestructive analysis of unexpected porosity or build defects in final parts, allowing a pass/fail decision for a part meant for a critical application. Various recent research efforts were aimed at qualification of AM processes, often for specific process parameters and material types. One such qualification was recently presented for AM of biomedical implants. ${ }^{59}$

A recent development is that witness specimens (test coupons) are manufactured in the same build as a critical component, and these witness specimens are then analyzed for defects at a high resolution. ${ }^{60}$ A statistical analysis of the detected porosity can be related to the porosity of the part itself and hence its mechanical properties. This allows the use of high-resolution microCT for prediction of a larger part's properties, and can be used effectively for builds containing fewer witness specimens than parts.
$\mathrm{X}$-ray inspection (2D) is a widely used and low-cost alternative nondestructive test method for the detection of flaws in castings, welds, and various metal processed parts. What is not widely known (or understood) is that typical microCT systems also have the ability to do $2 \mathrm{D} \mathrm{X}$-ray inspections, with the same or better quality than dedicated 2D inspection systems. Obviously, a full microCT scan provides a much clearer view of defects, with more quantitative data of the defects, and with a higher sensitivity and contrast. However, 2D X-ray imaging is almost real time, allowing for time and cost savings. There are obvious challenges to $2 \mathrm{D}$ imaging with complex parts as those produced in AM, due to different path lengths of material for the X-ray projection image. The possibility for combining $2 \mathrm{D}$ inspection of large numbers of parts with 3D microCT scans of selected parts allows for cost-effective inspections, especially for large production volumes.

Due to the need to ensure the detection capability of microCT for detection of small pores and cavities, various studies have created artifacts containing artificial cavities (seeded flaws). Such AM artifacts were produced containing cavities of varying sizes, and it was shown how microCT can accurately quantify the cavity sizes. ${ }^{61,62}$ Figure 2 shows one such artifact containing cubic shell cavities. It is demonstrated that the initial X-ray image, when contrasted properly, positively
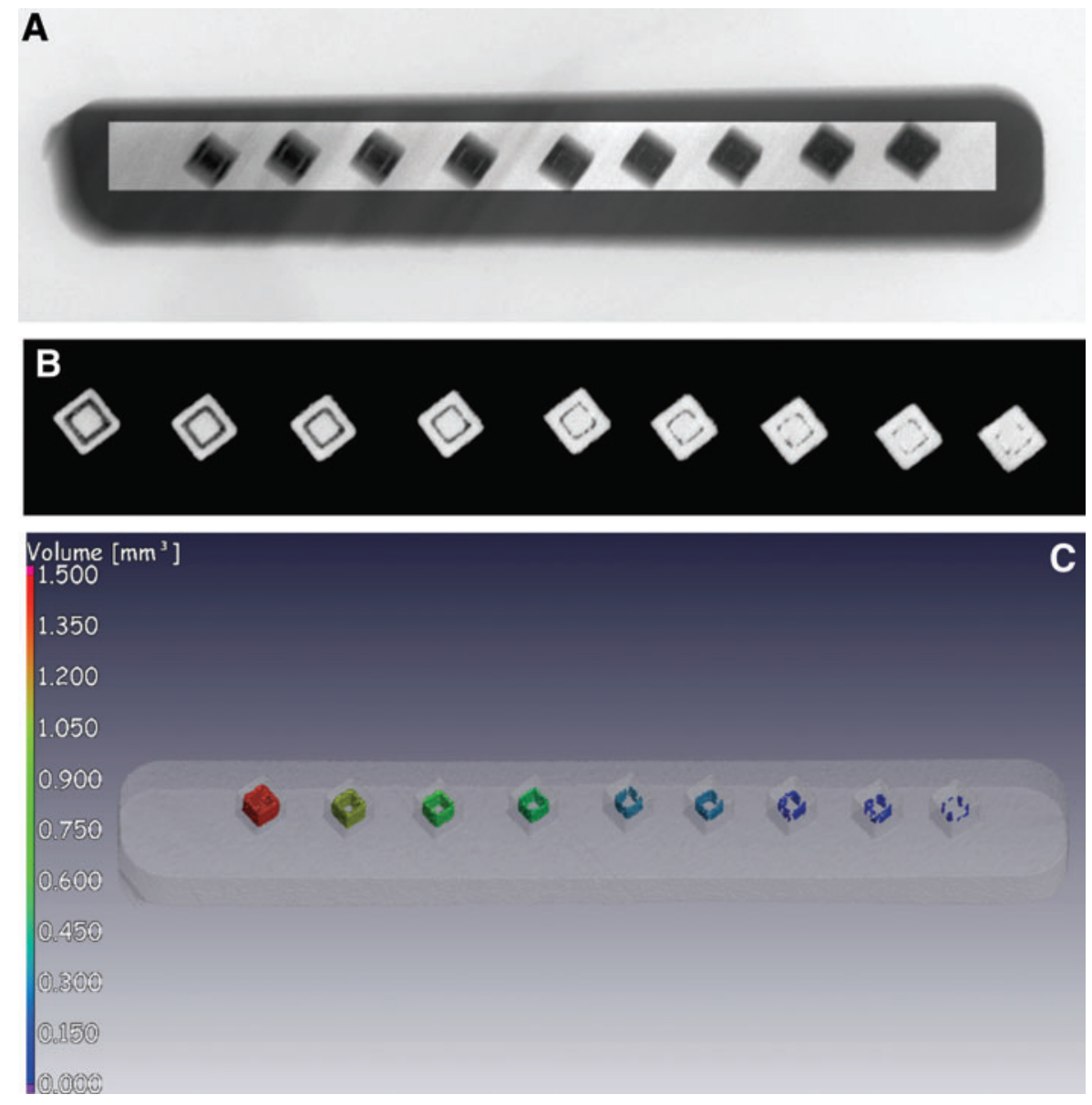

FIG. 2. X-ray and CT of LPBF Ti6Al4V ELI test sample with designed internal cavities of varying dimensions. The contrasted X-ray image (A) reveals the presence of all the cavities, while $\mathrm{CT}$ allows much clearer 3D visualization and quantitative assessment as seen in a CT slice image $(\mathbf{B})$ and $3 \mathrm{D}$ rendering $(\mathbf{C})$. LPBF, laser powder bed fusion. Color images available online at www.liebertpub.com/3dp 
identifies all the cavities, while the microCT data allow much clearer viewing of the cavities in slice images. A further porosity analysis allows quantification of pore volumes, cavity wall thickness, and more, including 3D visualization of the extent and $3 \mathrm{D}$ distribution of the cavities.
The X-ray image in Figure 2A was obtained with a typical acquisition during a CT scan-higher quality 2D X-ray images can be obtained with proper sample orientation and image averaging, within less than a few seconds. The subsequent microCT scan requires longer time investment in
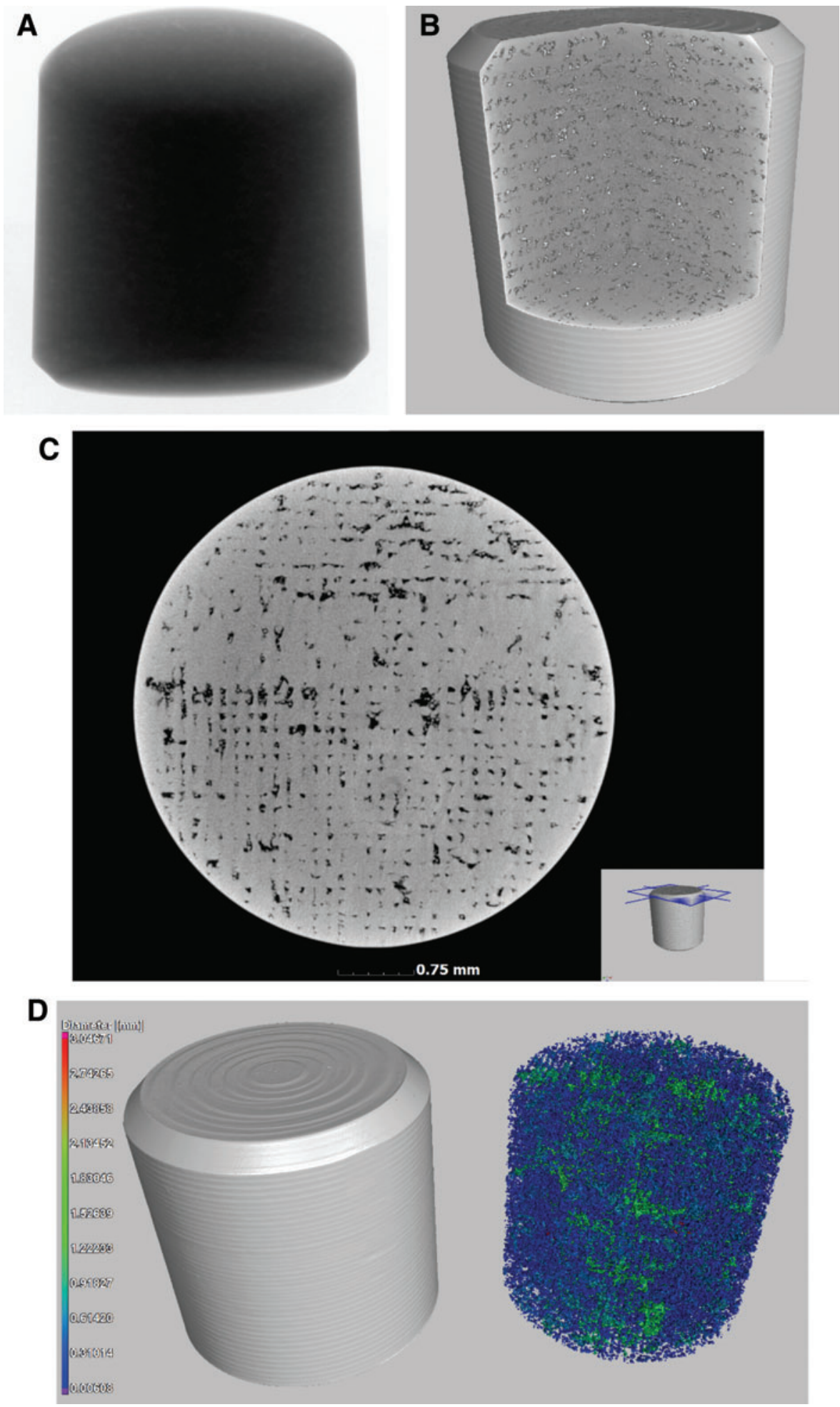

FIG. 3. Example of porosity analysis in a small cylindrical sample (3 mm diameter) produced by LPBF with nonoptimal processing parameters, leading to total porosity of $4.5 \%$. The porosity can be seen in an X-ray image (A) and visualized and quantified in different ways from CT data, shown in a 3D cropped view (B), CT slice image showing unmelted powder inside the pores (C), or a transparent view of the porosity analysis in 3D (D). Color images available online at www.liebertpub.com/3dp 
scanning and data processing to obtain results such as those presented in the figure.

An example of unexpected lack of fusion porosity produced by LPBF is shown in Figure 3. This sample was machined after $\mathrm{AM}$ to a cylinder of $3 \mathrm{~mm}$ diameter and height- the 2D $\mathrm{X}$-ray image is shown, followed by different microCT visualizations: a cropped 3D rendering, a slice image, and finally a $3 \mathrm{D}$ porosity analysis. This sample contains a total of $4.5 \%$ porosity, similar to some of the examples in Kim et al.,${ }^{42}$ and the 2D X-ray image already shows evidence of this porosity (white spots in the black area). The microCT data show in a cropped 3D view, lots of pores not visible on the surface, presumably due to the machining process the surface pores were closed up. The microCT slice image shows in black the pore distribution in a horizontal plane, or virtual cross section.

These cross sections can be analyzed in a stepwise manner without any further processing, to manually interpret the defects or to make 2D image analysis as is done with metallurgical microscopes. Finally, a porosity analysis highlights the pores in $3 \mathrm{D}$ by color coding based on size.

This analysis seems visually excessive, but this corresponds to $4.5 \%$ porosity. This excessive visual effect is due to the full 3D nature of the data and is not a misrepresentation. Therefore, it is important to realize that even small amounts of porosity are clearly visualized, and the statistical data are as important as the visual representation. In this case the
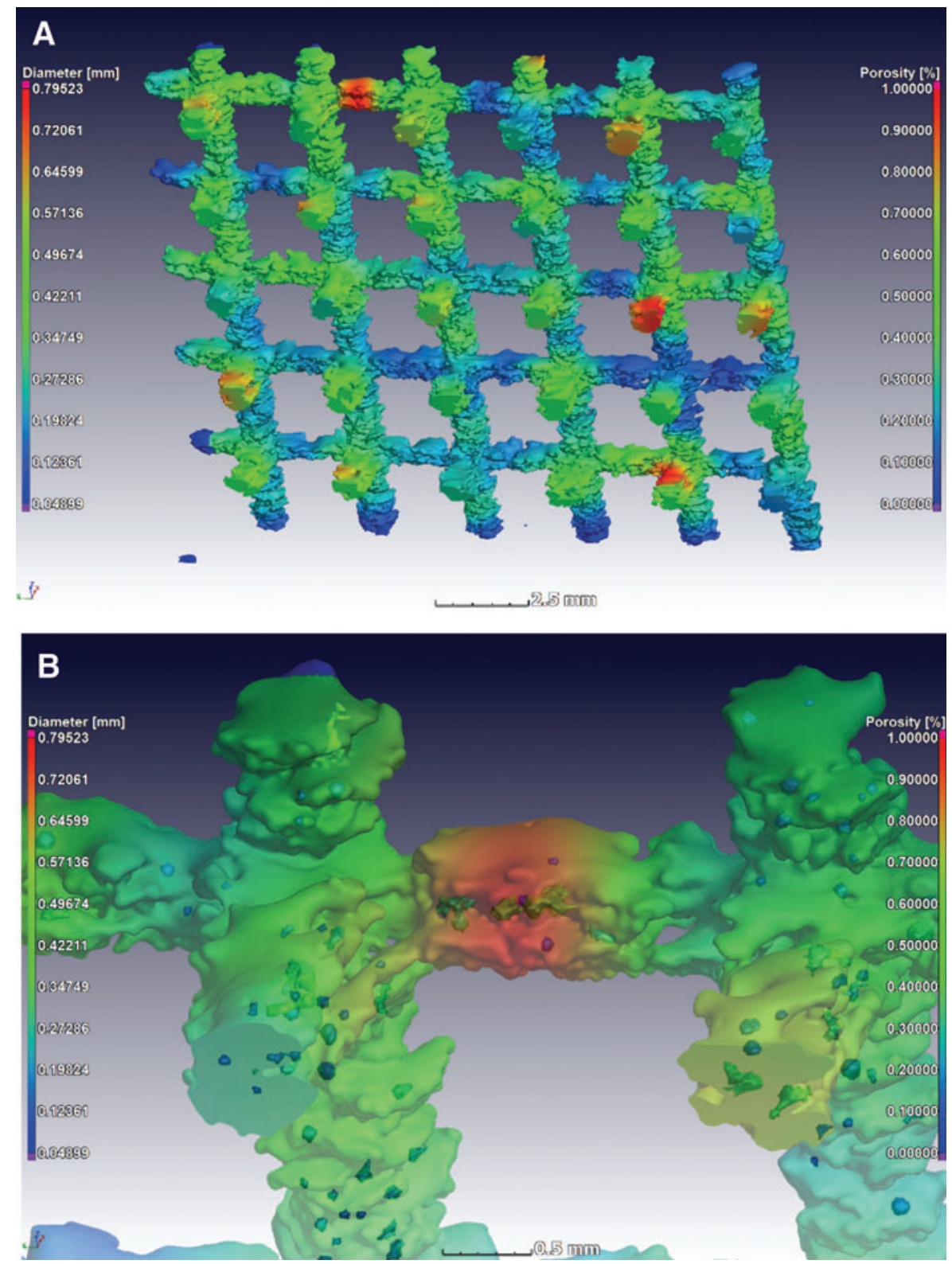

FIG. 4. Porosity inside the struts of a lattice, at an average level of $0.1 \%$. A local hotspot analysis shows areas of locally high average porosity (red parts $0.8 \%$ ), which will be weak points. A surface view with color bar relating to the local porosity is shown in (A). A close-up transparent view of the high local porosity in the top strut of (A) is shown in (B): this indicates which pores are responsible for the high local porosity, with their own color scale for their maximum diameters. Color images available online at www.liebertpub.com/3dp 
porosity is likely caused by lack of fusion porosity due to nonoptimal energy input for the chosen layer thickness. This conclusion is due to the trapped unmelted powder observed inside pores. Regularly spaced porosity (Fig. 3C) also indicates the possibility of too large hatch distance for the used scanning strategy.

Although "classical" porosity analysis by CT scans is useful, it is not only the size, quantity, or average level of porosity in a part that is important for mechanical properties of the part. Larger pores, more irregular pores, and those closer to the surface of AM samples are more detrimental to fatigue life, as they were found to be the main contributors to the stress concentration effect. $^{63-66}$ For this type of investigation, the pore distance from the edge of the part can be analyzed. Each pore can be classified according to its size, sphericity, location relative to surface, or even location relative to next nearest pore. Such classifications can be used to infer which pores are most critical, in continued studies of the critical pores in AM parts.

Similarly, clusters of pores in thin sections of a part can create local areas of high porosity, leading to weak points in the structure. It is possible to select regions and analyze the porosity over those regions only, but a new development is the automated calculation of porosity in equal-sized regions across the entire part. This method calculates porosity hotspots or areas of high local porosity and generates a color map distribution across a part, indicating areas of high local porosity within a predetermined region size. This is demonstrated in Figure 4 using an electron beam melted lattice structure as example. The red areas are those areas containing more than $0.8 \%$ porosity in local areas of $0.6 \mathrm{~mm}$ across, with a transparent view showing the pores responsible for the hotspots.

Metallic and nonmetallic inclusions are life-limiting failure mechanisms in structural materials, and cause reduced impact toughness and corrosion resistance. ${ }^{67,68}$ Such unwanted in- clusions, which are most likely due to impurity in the used powder AM system, can be detected by microCT. One example of this is shown in Figure 5, which is a microCT slice image of a compact tension LPBF Ti6Al4V specimen. Clearly visible are isolated pores (black) and many small white spotsinclusions. These inclusions can act as stress concentrators and affect the fatigue life and crack formation.

Also visible are some typical image artifacts, which is due to the sample scanned perfectly horizontally. In this case, the choice was made to scan in this way to improve the resolution of the middle section, as the interest in this case was to obtain high-resolution images and porosity information of the region below the crack notch (which is not yet machined into the sample). This region does not contain artifacts. It is important to note that not all types of inclusions can be detected by microCT and the detection capability scales with resolution of the scan, as in porosity analysis.

\section{Volumetric density measurement}

Despite the capabilities of microCT to image and quantify porosity and defects, AM parts are still typically subjected to Archimedes tests to measure their average volumetric density. This test is simple and fast (and low cost). However, there are some potential problems with this method:

- Air bubbles can attach to the surface especially due to the rough and irregular surface, resulting in larger volume measurement and hence lower measured density.

- Channels or flaws connected to the surface can be filled with water, resulting in a smaller volume measurement and hence a higher measured density.

- Inclusions can potentially increase the measured mass and hence measured density.

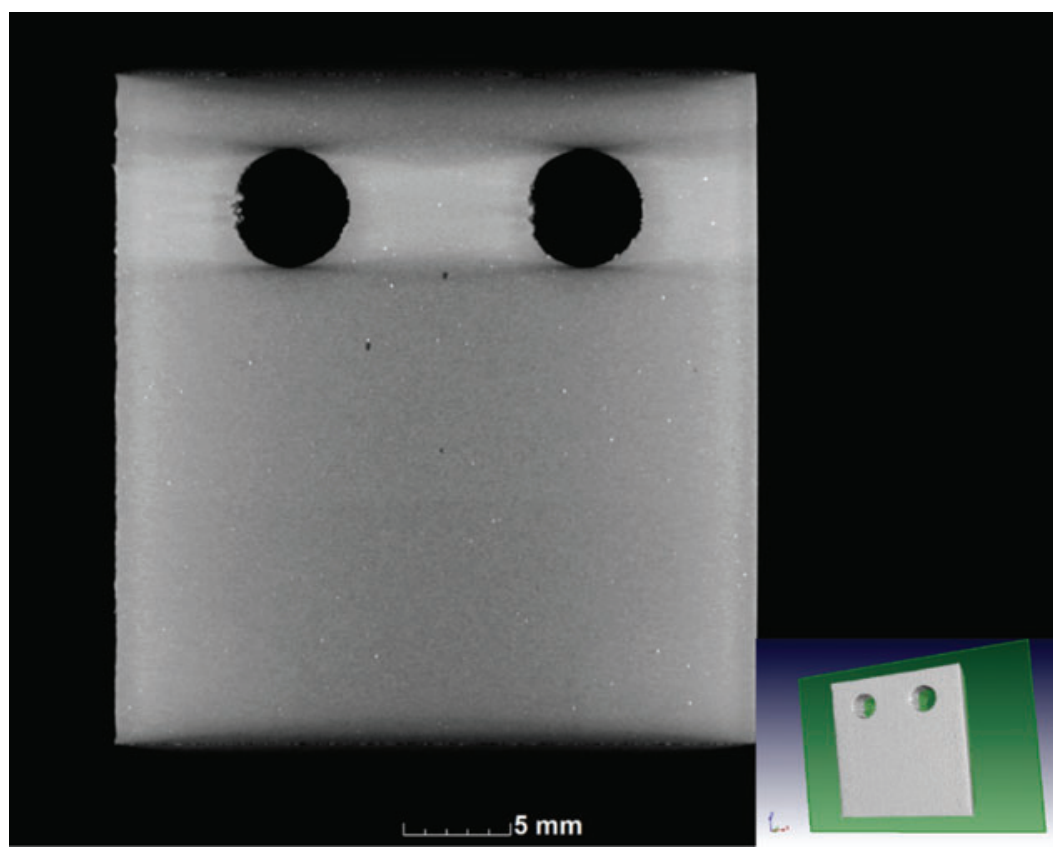

FIG. 5. Isolated pores in a compact tension specimen, before the notch being machined into it (between the holes). Black spots indicate small isolated pores that can affect the crack growth in a fatigue test. Also visible are bright spots, which are impurities (inclusions), which may also act as stress concentration points and hence affect the fatigue crack growth behavior. Color images available online at www.liebertpub.com/3dp 
- Major flaws such as layer defects, keyhole or gas pores take very little volume (e.g., $<0.01 \%$ ) and hence do not make much difference to the measured density but can still be very important if they are clustered or layered, or have some nonrandom distribution.

- A material density is assumed, which can be incorrect for alloys with varying compositions.

While porosity analysis by microCT is well known as shown in the previous section, it is not so well known that the entire part volume can be accurately determined-this combined with the part mass provides a good measure of the average part volumetric density. This can be useful to complement or overcome some of the abovementioned issues with traditional density measurements. Theoretically this is better than porosity analysis using microCT image analysis, as it is less prone to detection capability issues of the microCT instrument (as small porosity may be missed in some scans while an accurate part volume is still measured).

This CT-measured volume of the part can overcome the problems with air bubbles and open porosity/channels, while additionally visually confirming the presence or lack of inclusions and small porosity or defects, that is, identifying the causes of density problems. The accuracy of the volumetric measurement of the part is limited by the scan resolution only, requires images free of artifacts, and requires an accurate subvoxel surface edge determination. It is envisaged that this be useful primarily on test coupons and small samples, due to the accuracy of the surface data required.

Finally, it is also possible to use CT with calibrated density samples, scanned together with an unknown sample to calculate its density based on grayscale alone-this requires low-resolution scanning and is done similar to the process described in du Plessis et al. ${ }^{69} \mathrm{It}$ is important to realize that the same material is required with varying densities covering the expected range of the unknown sample, which is sometimes difficult to acquire or produce. Yet, this may be very useful for fast relative density measurements.

\section{Dimensional measurement}

A widely used quality control method in AM is the dimensional assessment or metrology of produced parts. Much work is done using traditional metrology methods (e.g., coordinate measurement machines [CMMs] and optical measurement systems), but these are limited to exterior surfaces. X-ray microCT is increasingly being used for precision coordinate measurement applications in various industries, as the method is refined and sources of uncertainties better understood - this is summarized in a recent article comparing CMM and microCT demonstrating less than $5 \mu$ m deviation in most cases. ${ }^{70}$

It is also the only method capable of measuring dimensions of complex parts containing internal surfaces and lattice parts. One of the simplest dimensional assessments is checking for powder or melted material inside complex parts, for example, in lattices or inside channels not visible, which can cause blockages or affect the performance of the parts in some way. This can be done by simple viewing of CT slice images from a reasonably fast microCT scan, such as the example shown in Figure 1, where powder remains inside the part and is visible in the CT image as lower density (less bright) material.

MicroCT-based dimensional measurements (metrology) require higher quality scan data and geometrically calibrated systems. ${ }^{71}$ Recently, dedicated metrology CT systems have been introduced, which are temperature stabilized, and calibrated test parts are scanned before every scan to validate the dimensional accuracy to a high level of confidence and provide traceability of the results.

However, the scan setup and microCT scan settings play a very important role in generating high-quality data (highcontrast, sharp material edges and low noise, lack of artifacts). As the data quality improves so does the accuracy of the obtained surfaces and hence dimensional measurements based on these surfaces. Finally, the determination of the surface at subvoxel accuracy is possible using 3D interpolation of gray values, which is only useful when no image artifacts are present and signal to noise ratio is high. For obtaining highquality data, some guidelines are presented in Refs. ${ }^{11,12}$

Dimensional measurements can take various forms: simple linear measurements in aligned CT slice images, wall thickness analyses of produced part walls, or complete part surface comparison to computer-aided design (CAD) model or to another part. Different process parameters and scanning strategies in AM can result in the real dimensions of manufactured parts deviating from the geometrical characteristics prescribed by the CAD model.

For example, LPBF cylindrical samples produced at similar process parameters, but two scanning strategies-single scan and rescan-had different dimensions. It was found that samples fabricated by a single scan strategy had about $60 \%$ of surfaces less than the prescribed CAD surfaces, while rescanned samples had about $75 \%$ of surfaces more than the CAD ones. ${ }^{39}$ Geometric tolerances and deviations from CAD design of microchannels were analyzed by CT scans in Snyder et al. ${ }^{72}$ On the base of this deviation data, scaling parameters can be introduced to precompensate for deviations resulting in a more optimized final part dimension, taking into account shrinkage behavior of the material, part geometry, and most building and scanning strategies.

Figure 6 shows an example of CAD variance analysis (compare actual part to design file) of an LPBF Ti6A14V ELI adult human facial implant produced in CRPM, Central University of Technology, Free State, South Africa, with an EOSINT M280 (EOS Electro Optical Systems Ltd.) at manufacturer-prescribed process parameters and scanning strategy. The fabricated part with support structures deviated from the CAD model in that some areas were lower than $0.5 \mathrm{~mm}$ below the CAD design (blue-purple) and other parts were above the CAD design up to $0.5 \mathrm{~mm}$ (red). This indicates slight warping during processing, which can be problematic for custom human implants and resulted in this part being rejected. After a comprehensive study of the building strategy and CAD variance analysis, a new part was built in a different orientation and including additional bars to prevent warpage during processing.

Another complex and specialized dimensional measurement capability for microCT, which is starting to be utilized more often, is the analysis of AM lattice structures. Such lattice structures are widely used in AM parts for lightweighting, and in medical implants for bone ingrowth. There is significant development in the AM community to effectively use lattice structures, making use of topology optimization. ${ }^{73}$ The 3D analysis capabilities of microCT for this type of part originate from biomedical bone structure analysis, ${ }^{74}$ but are equally suitable to lattices. 


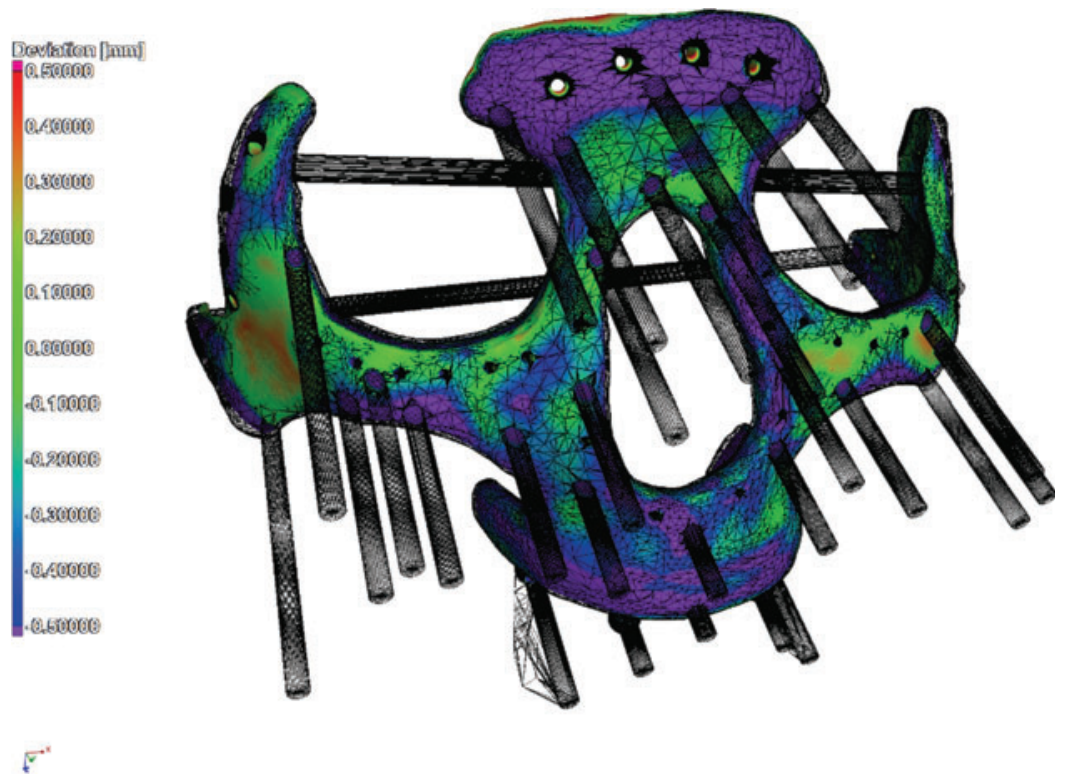

FIG. 6. CAD variance analysis of facial implant built by LPBF-CAD file mesh includes supports. Blue areas are smaller than design, red are larger. CAD, computer-aided design. Color images available online at www.liebertpub.com/3dp

Lattice structures can often have thin struts that can be problematic to build accurately and might contain unmelted material, particles attached to the surface, or other imperfections difficult to remove from inside the lattice. A relatively large lattice structure with mean strut thickness of $0.9 \mathrm{~mm}$ is shown in Figure 7; in this case the color coding shows the thickness at every point. The same lattice contains entirely open connected porosity, which cannot be quantified in the same way as isolated closed porosity. For this type of open porosity, a maximal-sphere algorithm is used similar to the strut thickness measurement, but instead used to quantify the pore spaces. This allows the pore size determination as $2.5 \mathrm{~mm}$ in this case for the bulk pore spaces as shown in Figure 7 using a 3D view and slice image.

While some analyses such as those above for lattices can be time-consuming, there are three cost-effective solutions to microCT-based inspection of lattice structures and struts:

(1) simple viewing of the slices allows identification of potential problems and simple and fast linear measurements;

(2) basic morphological analyses such as total material volume, pore space or fraction, and material surface area. These simple measurements allow calculation (based on volume and surface area) of estimated values for strut thickness and spacing (termed trabecular thickness and trabecular spacing according to the original biomedical use for bone analysis ${ }^{75}$ ). This method is faster, but a good estimate, compared with the maximal sphere method that measures directly the thickness of the strut at every point as shown above and described in more detail in Hildebrand and Ruegsegger. ${ }^{74}$ However, this is a stereological method and is based on surface area, which might be significantly higher for some rough surfaces as found in AM parts, therefore it should be used carefully;

(3) extraction of STL file from the microCT data allows for basic analysis in any other CAD viewing software, and allows one to keep a record of the data in a format widely used in the AM community, requiring no significant data capacity or specialized software tools.

\section{Deformation or $4 D$ microCT}

One particularly useful aspect of microCT is the ability to monitor changes in the same part, especially after some change or deformation-this can be performed in situ or ex situ. In situ experiments are possible by the use of specially designed stages allowing compression or tensile testing in the microCT chamber, and similarly designed temperature stages have been used.

For in situ experiments, a sample is typically scanned unloaded and loaded to analyze deformations and identify the location of first failure. For ex situ experiments, the sample is first scanned, then subjected to mechanical testing up to a certain point, the test stopped (e.g., at $6 \%$ elongation), and a new scan is performed on the sample. In either case, this type of "time lapse" CT is termed 4D CT and provides a powerful new tool to identify the material response to different processes.

The method has been used to monitor deformation changes in AM parts subjected to static loading tensile tests, in attempts to visualize the effect of porosity on fracturing. ${ }^{76}$ In this work, a unique analysis was devised through which the tensile necking behavior was visualized and quantified using a CAD variance analysis between the deformed sample and a best-fit cylinder to the original shape. In this way, the asymmetrical necking behavior could be visualized.

The same work was extended with a single sample manually aligned between original and fractured sample, as shown in Figure $8{ }^{55}$ This manual correlation of pores is challenging as the pores are very small, and new pores emerge above the scan resolution due to the elongation and fracturing occurring. This type of manual volumetric correlation can allow investigations of root cause pores resulting in fracture, for example. Interestingly, the necking and fracture do not occur in the area of highest porosity in this sample (the bottom part). This indicates 

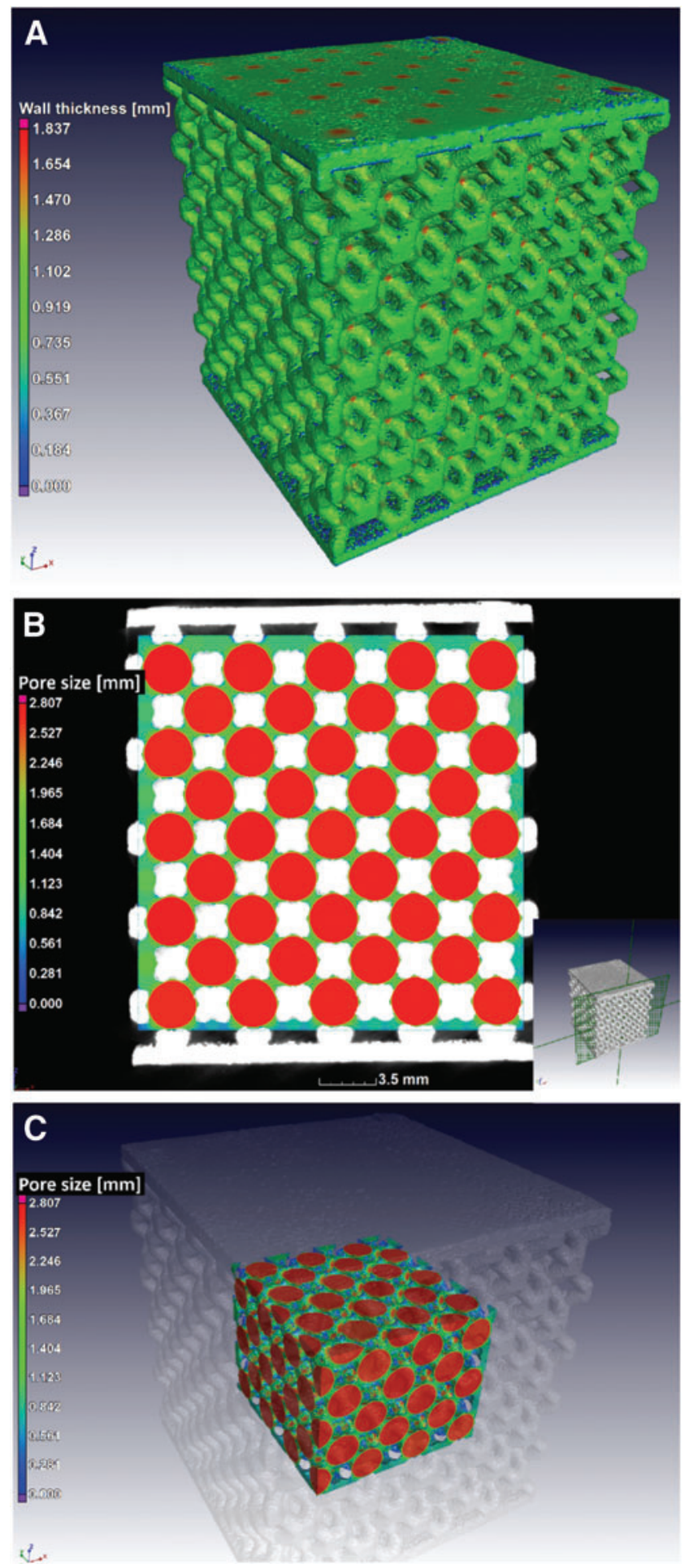

FIG. 7. Strut thickness and pore space analysis in lattices. A strut thickness analysis is shown in 3D in (A), a slice image shows the pore size analysis of the open porosity in $2 \mathrm{D}$ in (B) and in $3 \mathrm{D}$ in (C). Color images available online at www.liebertpub.com/3dp

that these small pores are not critical for static strength of parts. In this case, the microstructure played the dominant role in the yielding and failure location.

Generally, 4D CT can be combined with digital volume correlation to provide full-field strain maps. ${ }^{77}$ In situ deformation and temperature changes can be followed using specially designed stages, for example, as shown in Refs. ${ }^{78,79}$ Other applications can be to monitor the efficiency of pore closure in HIP treatment and the effect of heat treatments on pores. $14,52,53,80$

\section{Surface roughness or topography}

AM parts require surface roughness assessment, but traditional tools such as tactile and optical profilometers and laser scanners work only on the exterior surfaces of parts, and often only in lines or on flat surfaces. ${ }^{81,82}$ It was shown previously that individual line profiles in $\mathrm{CT}$ images can be related to surface roughness, especially useful for complex parts such as struts in lattices. ${ }^{83,84}$

Recent advances in image processing of high-quality microCT scans have demonstrated that it is possible to measure surface topography of AM parts generally, ${ }^{85}$ also for internal surfaces of complex parts. ${ }^{49,72,86}$ The term topography is used since the resolution does not allow for roughness measurements near or below the resolution of the scan, and the method is therefore different from typical traditional surface roughness measurements. However, the advantages of allowing for measurement of internal surfaces and the surfaces of complex parts such as lattice structures make it extremely useful for AM, especially for internal surfaces of parts. The values for internal surface topography using microCT compare very well to the same surfaces measured using traditional noncontact surface measurement methods, where the internal surface was exposed for the optical measurements. ${ }^{86}$

The practical limitation for microCT-based surface characterization is the resolution and part size: the voxel size is limited by the part size and hence the method is only suitable for reasonably rough surfaces, or small parts (or both). The above studies focused on flat surfaces, but it is also possible to characterize nonflat surfaces (curved surfaces or geometrical shapes). This can be done by fitting a geometric shape to the part (e.g., a cylinder as in Fig. 9) and measuring the deviation of the actual surface from the geometric shape at every point, similar to a CAD variance.

\section{Simulations}

The use of microCT data as a basis for simulations is an exciting possibility that has not been widely explored yet. Unlike simulations based on design geometry of parts, the actual parts, including defects, surface imperfections, and build errors, can be simulated, providing a theoretically more accurate prediction of the properties of the part. This can be useful for two reasons: first, the actual effect of a defect on the resulting mechanical properties can be assessed, assisting to make pass/fail decisions on the use of a part; and second, the effect of defects can be studied and correlated with various mechanical tests on the same parts.

This latter route has been applied in a study of tensile testing of cast Ti6Al4V containing large pores up to $4 \mathrm{~mm}$ in diameter. ${ }^{59}$ In this study, the static yield strength was correlated with pore size, and fracture occurred predominantly at the largest pore, but yield strength was not severely affected (it was still high despite the pores). This was most likely due to the rounded shape and central location of the casting porosity; pores closer to the surface are expected to contribute to much higher stress concentrations, which will result in a decrease in strength of the part. 


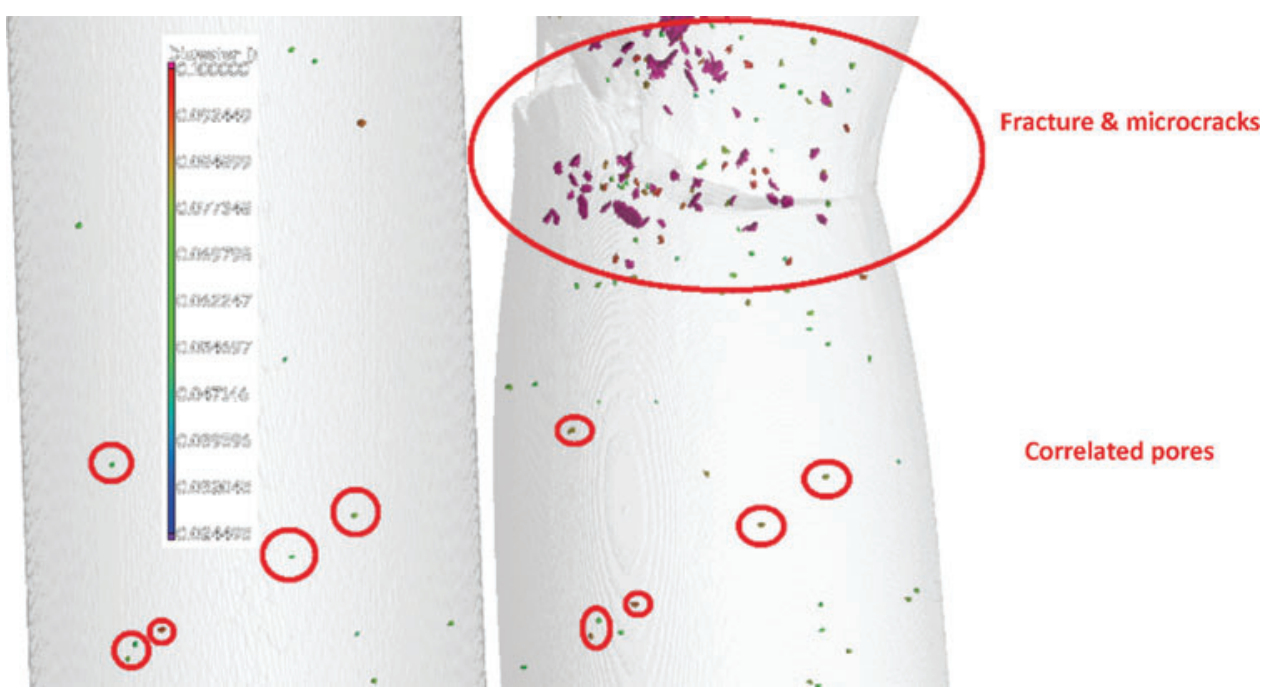

FIG. 8. As-built LPBF Ti6Al4V ELI tensile sample deformed showing necking, fracturing, and correlated pores. Color images available online at www.liebertpub.com/3dp

Perhaps more important than static strength is the effect of pores on fatigue life of a part. This was investigated for electron beam melted fatigue samples by microCT coupled with simulations in Tammas-Williams et al., ${ }^{87}$ where it was found that the crack initiation defect was often not the largest, the proximity to the surface is often more important. In this work, as in much prior work on microCT-based simulations, the typical workflow requires the images to be converted to meshes suitable for mechanical or other simulations in dedicated simulation packages.

Recently, structural mechanics simulations have become possible directly on voxel data, using an immersed boundary finite element code incorporated directly in voxel analysis software. This simulation method assumes linear elastic material properties and small deformations. It has been used in the abovementioned study of stress concentrations around casting pores subjected to tensile tests, ${ }^{59}$ and, more recently, it was applied to analyze stress concentrations in AM brackets, predicting which parts have longer fatigue life. ${ }^{88}$
Similar concepts for predicting the consequence of a defect visualized by X-ray CT were investigated for an improved understanding of fatigue critical pores in AM parts in Siddique et $a l .{ }^{64}$ It was found that the stress concentration factors for pores near the surface are very high, and suggestions were made for a remelting strategy in these areas.

An example of a direct voxel-based simulation of static loading of a 25-mm-wide LPBF Ti6Al4V lattice structure is shown in Figure 10 in CT slice images with simulation (left) and after compression to first failure (right). This clearly demonstrates the use of the stress simulation as the failure location corresponds to the high stress region. If there were pores in the high stress regions, this would be exaggerated and create an even higher stress region. Besides visualizing the locations of high Von Mises stress, and potentially correlating this with imperfections in the part or porosity clusters, the method also provides statistical information on regions of high Von Mises stress (hotspots), and similar information on deformation, which

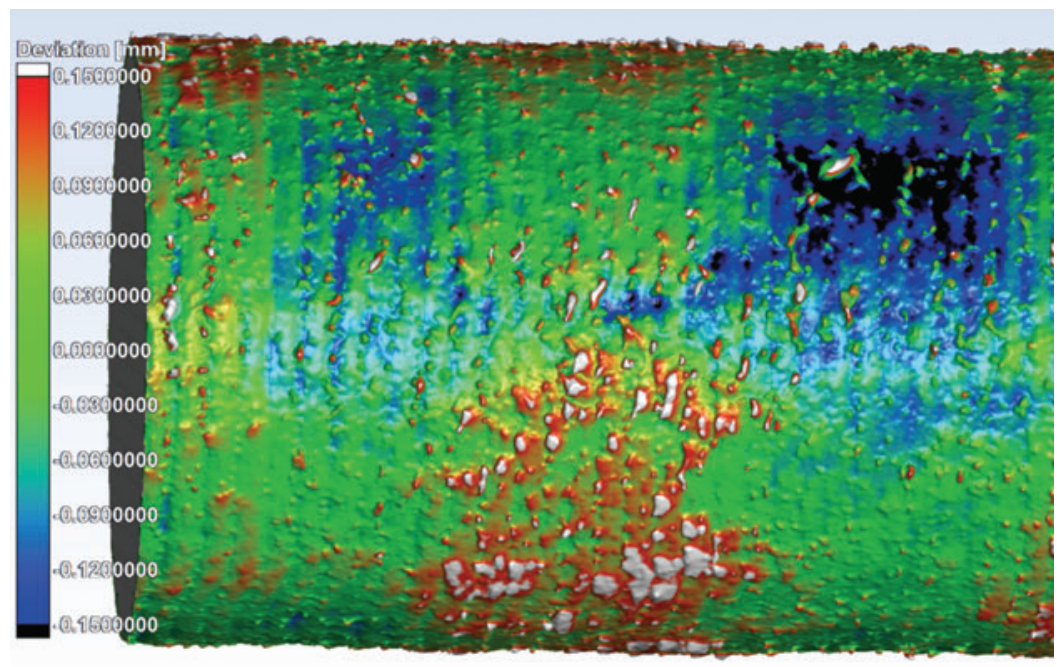

FIG. 9. Surface roughness/topography analysis of AM part on a cylindrical geometry. AM, additive manufacturing. Color images available online at www.liebertpub.com/3dp 

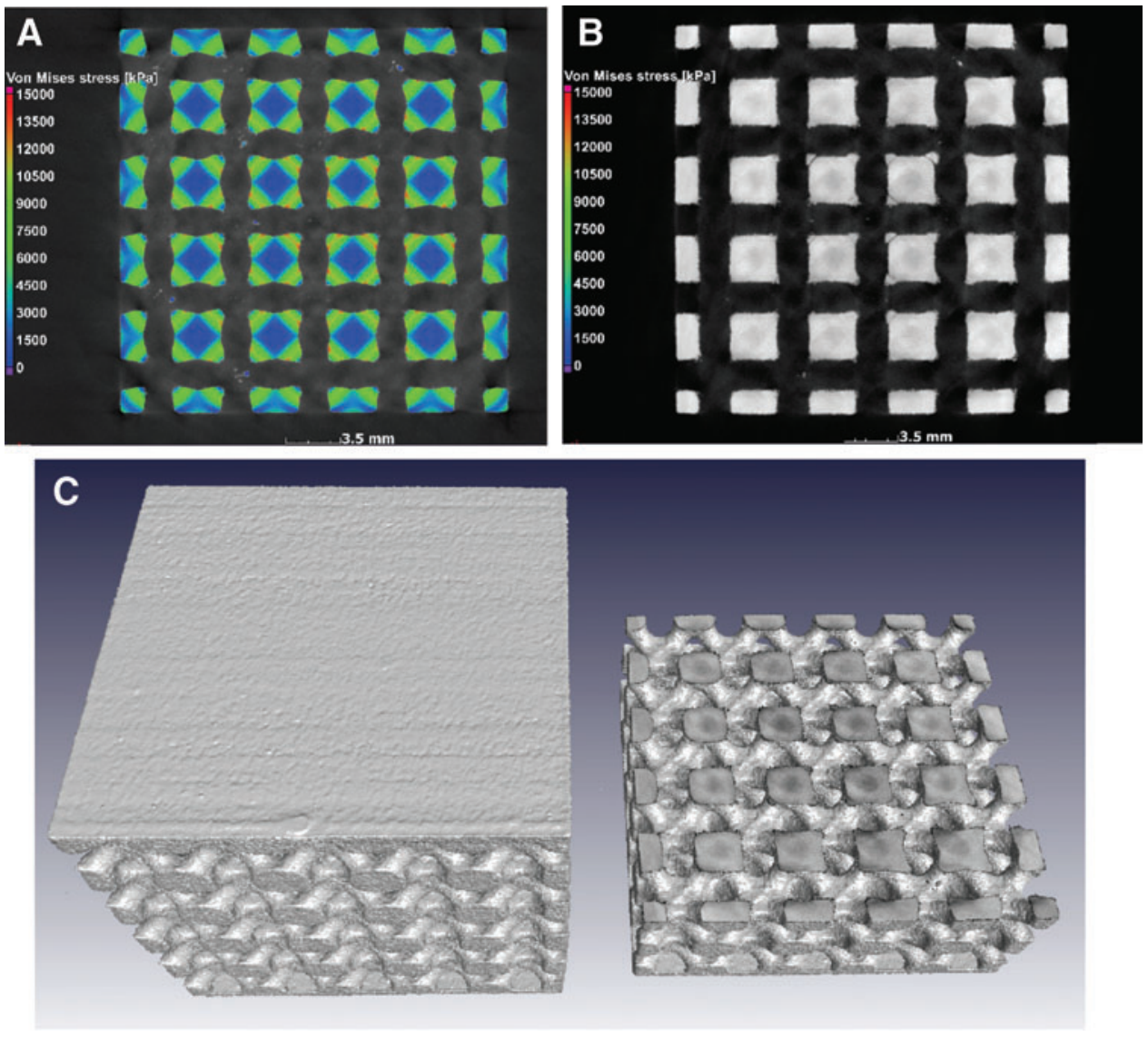

FIG. 10. Von Mises stress from simulation of compressive loading on microCT data of 25 mm LPBF Ti6Al4V ELI lattice sample-viewed in microCT slice images before compression (A) and microCT image of compressed sample after first failure (B). This shows cracks at the location of high stress in the simulation before compression. ${ }^{89}$ The $3 \mathrm{D}$ rendering $(\mathbf{C})$ shows in a cropped view the location of the cracks. Color images available online at www.liebertpub.com/3dp

can be used to calculate an effective elastic modulus of a lattice structure, for example.

The lattice shown here is $50 \%$ dense and the simulated elastic modulus is $29 \mathrm{MPa}$ on the real sample, compared with $20 \mathrm{MPa}$ obtained in the experiment (average of three replicates), and the location of first failure corresponds to high Von Mises stress regions in the simulations-this work is submitted for publication elsewhere. ${ }^{89}$

\section{Multiscale CT and fast scanning}

In a study of the use of microCT for quality control of an AM medical implant, ${ }^{58}$ multiscale microCT scans $(100,75$, 50 , and $25 \mu \mathrm{m}$ ) indicated the ability to detect progressively smaller porosity at higher resolution. In this work, a reference sample was used to validate the detection capability, in the same scan. This is a similar concept to using a coupon sample with seeded flaws, as has been mentioned. Poor resolution results in faster scan times due to less angular projection images required for a good reconstructed image, which is useful for cost-effective use of the method. Similarly, each acquired image can be obtained in shorter times and with less averaging of images, also increasing the scanning speed. In a multiscale microCT study of porosity in concrete, it was shown that even 5 -min scans can produce useful porosity information. ${ }^{90}$

This method of fast scanning reduces the ability to detect the smallest pores by adding to noise levels, but the largest (and most likely most important) defects are still identified. In a similar methodology, many samples can be scanned at once, as demonstrated in Guelpa et al. ${ }^{91}$ However, scanning many samples at once has numerous drawbacks: the resolution is poor, higher penetrating power is required for many dense samples, significantly reducing data quality (noisy data), and the scan data also contain various parts and therefore require additional processing to separate the parts virtually (with the possibility to mix data).

Therefore, fast individual scans are suggested for AM parts, as a cost-effective quality control tool. This can be used in the form of "scout scanning" to identify potentially defective parts, and when something is observed, a high-quality scan can be conducted. The same can be done with X-ray inspections instead of fast scanning, but for complex parts such as AM parts, and with the types of defects (e.g., thin layered defects), this is not suggested.

The other extreme involves slower scans due to extended acquisition times and more acquisition images with additional image averaging. The adjustments can significantly improve the quality of the obtained images, which will improve the detection limit of small features. It is also possible to scan a small section of a part at a higher resolution than that possible on a whole part. This could be required to test a small critical section of a larger part, such as a thin wall of a medical device, for example. In this case there are two options that are useful. First, region of interest scanning is possible, that is, the field of 
view is limited and the rest of the material rotating around this area being imaged. Usually this is done in combination with full-part scans. Another option is to scan elongated parts in sections and stitch them together into one large volume.

Moving the detector horizontally allows wider samples to be scanned increasing the obtainable resolution for the same part size. This allows high-resolution imaging of the entire sample. Some systems also allow helical scanning, which eliminates the need for stitching and removes some types of edge artifacts, allowing higher magnification at high quality. Stitched doublehelical scans offer a combination of these advantages for higher resolution. Further hardware developments are in progress using higher resolution detectors to improve the obtained resolution of microCT scans, for the same size part. Improved resolution on large parts is practically limited by computing resources available, as the full volumetric data set typically needs to be loaded to memory for analysis - this requires more than $20 \mathrm{~Gb}$ memory even for typical data sets.

\section{Powder analysis}

The analysis of metal powders has recently become possible using high-resolution microCT, as demonstrated in Refs. ${ }^{92,93}$ The usefulness of this over conventional laser diffraction or microscopy is primarily that internal pores can be easily identified in powders, which might be trapped in the meltpool during the AM process. ${ }^{45}$ It is also the only method through which the real sphericity, volume, and surface areas of particles can be determined, which can all be important parameters for ensuring flowability and eventually quality of the powder bed.

In the first demonstrations of metal particle analysis by microCT, sample preparation was required for loading particles in such a way to prevent them from touching, making digital analysis simpler. However, new algorithms have become available recently allowing image filtering using a nonlocal means filter, and when this is coupled with a newly developed foam structure analysis (digitally separating touching components), a complete powder analysis can be made of powders loaded and scanned in a pipette tip. A similar process can be followed using a watershed segmentation process in other software packages. This is demonstrated in Figure 11 for Ti6Al4V particles, and the method is applied and described in more detail in Rozendaal et al. ${ }^{94}$

\section{Multimaterials}

Most work to date using microCT in AM has focused on single materials. It is possible to analyze multiple materials in a part, but the quality of the resulting images may vary depending on the materials investigated. The example shown in Figure 12 is a special case of an interface between copper and steel - the part was manufactured in one piece by AM and the interest was to analyze the interface and visualize a possible
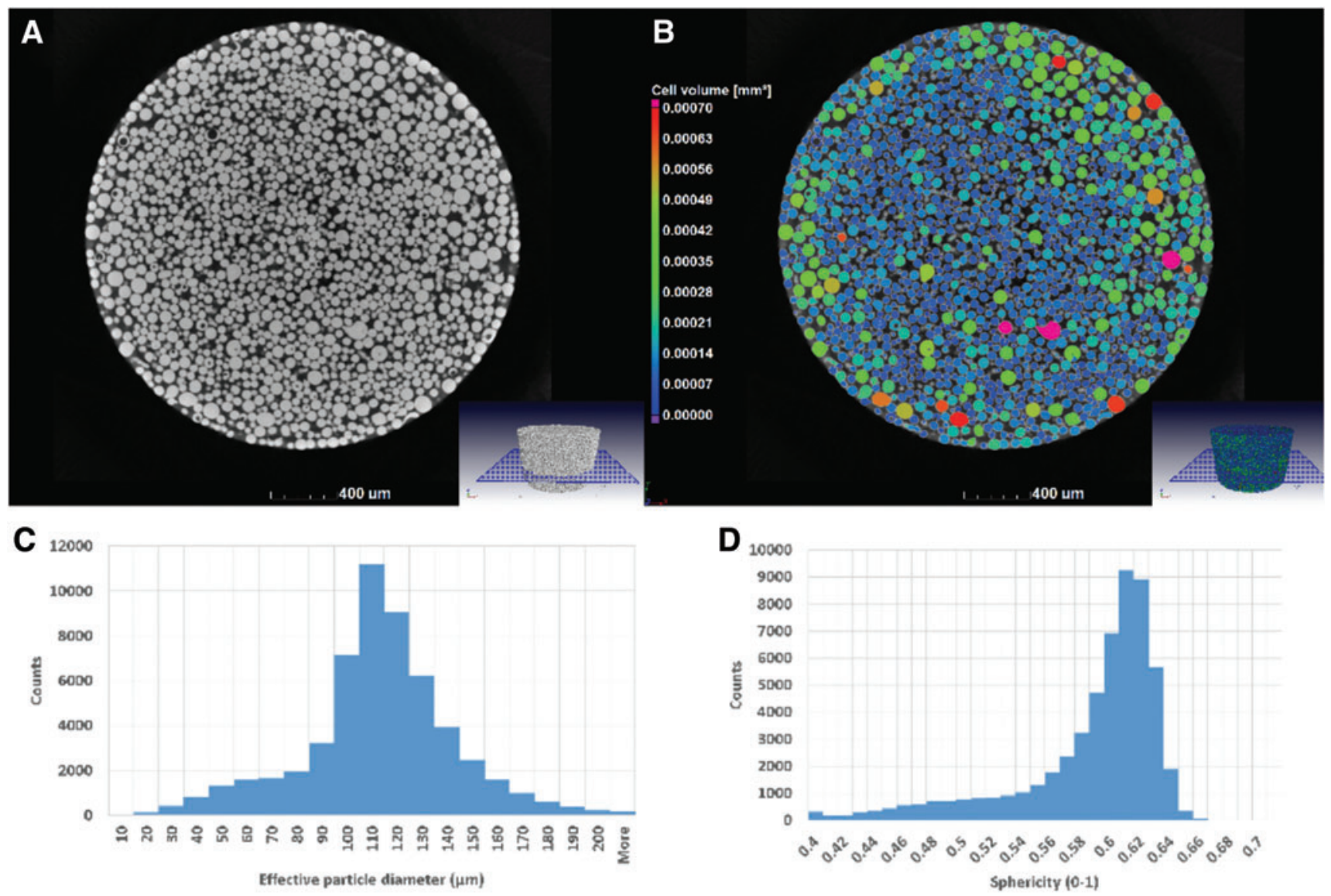

FIG. 11. Metal powder analysis by microCT, indicating the CT slice image (A), the color-coded size analysis (B), extracted information on particle size distribution (C), and sphericity distribution (D). Color images available online at www .liebertpub.com/3dp 


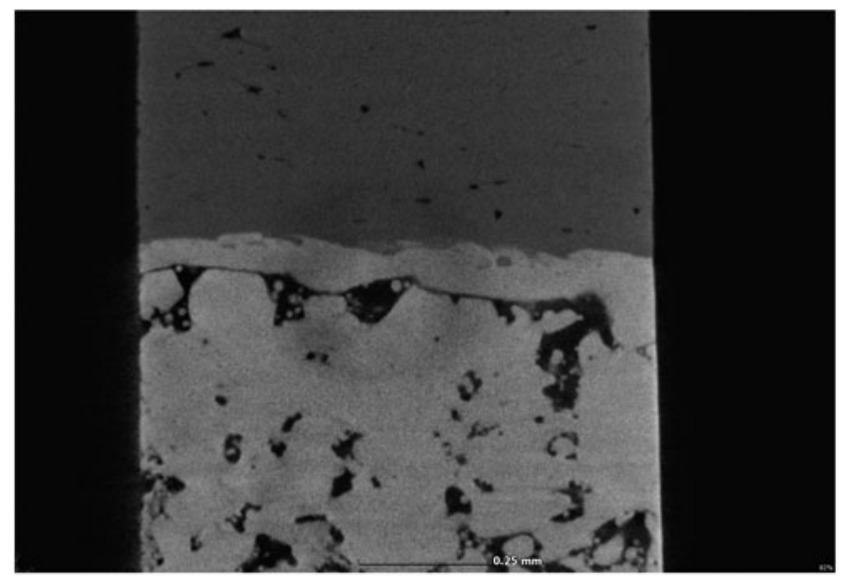

FIG. 12. Multimaterial interface shows good interface region between steel (top) and copper (bottom). Due to nonoptimal process parameters, the sample reveals a high porosity. The sample size is $1 \mathrm{~mm}$ wide, with scan at submicron voxel size $(0.9 \mu \mathrm{m})$.

region of intermediate density. Clearly the process parameters require some optimization, as lack of fusion porosity is observed, but the interface itself is free of porosity with some mixing observed in a wavy pattern. This is a small field of view of less than $1 \mathrm{~mm}$, as it is a nanoCT scan to obtain best possible resolution on the interface.

In more extreme cases such as steel (very dense) and aluminum (very light), the contrast on the steel will be good, while the contrast on the aluminum will be poor-this is much like attempting to view something while looking into the sun. It is therefore important to realize that the detail detectability is affected by the material in the scan volume. This is something that must be considered, for example, for light metal parts containing denser parts such as steel screws and bolts-it is best to remove these for best quality imaging of the aluminum part.

In another example of the multimaterial capabilities of microCT, the image in Figure 13 shows the distribution and sizes of unmelted Mo particles in a single laser melted track. This work investigated the in situ alloying of Mo particles in Ti and is reported in detail in Dzogbewu et al. ${ }^{95}$ The interest is to see how much of the Mo is melted, how these particles are distributed in the track, and if there are variations in density across the track. The particles seen in the image are unmelted and it was found that twice as much unmelted Mo particles are present in tracks melted at $150 \mathrm{~W}$ compared with those melted at $350 \mathrm{~W}$.

\section{Limitations}

One of the first and most important limitations is the part size, especially for metal parts. When a part is too big, penetration of X-rays becomes an issue requiring high scan voltages, beam filtration, and resulting loss of quality in the images. This can often result in lack of detection capability on small pores and can lead to some edges of the part being less or more bright than others in the CT data, making a good surface model impossible, or very time-consuming, to correct using image processing methods. This is especially true of denser metals, and objects larger than $100 \mathrm{~mm}$. The data can still be used for viewing for major defects but any more advanced analysis becomes much more challenging.

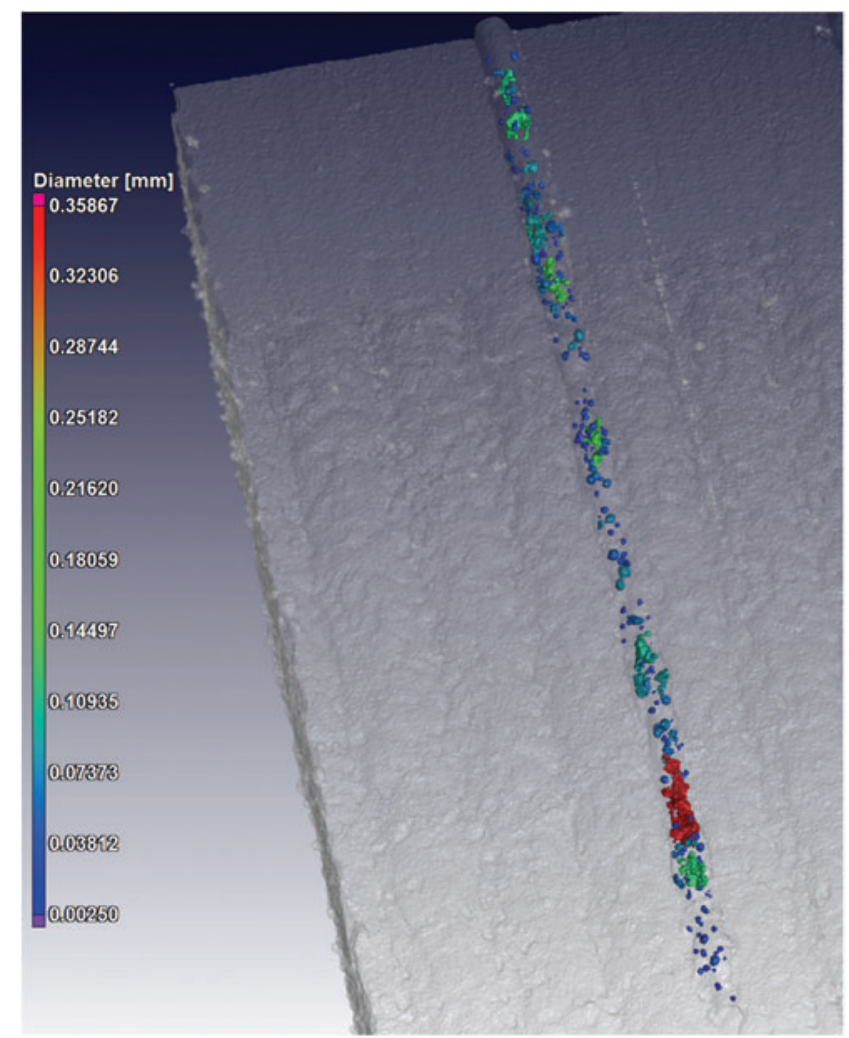

FIG. 13. In situ alloying of Mo analyzed by microCT, showing the distribution and extent of unmelted Mo particles in the single laser-melted track. Color images available online at www.liebertpub.com/3dp

One way to overcome this is to use higher voltage systems, or lots of beam filtration, and optimize scanning parameters for the object of interest. Beam hardening correction is crucial as a part of the reconstruction process, for large and dense parts.

Related to the part size problem is the required resolution that scales with part size. The smaller the part is the better the possible resolution that can be obtained. The "general rule" for typical geometrical magnification microCT is that the best possible resolution is 2000 times smaller than the widest part of the sample. For loading samples at angles and eliminating some kinds of artifacts, as is required for advanced analyses, this reduces to a factor 1000 .

For typical microCT scans at high quality, parts therefore need to be in the range of $10-100 \mathrm{~mm}$ (for voxel sizes 10 $100 \mu \mathrm{m})$ and for specialized high-resolution systems allowing submicron voxel size, typical sample sizes range from 0.5 to $10 \mathrm{~mm}$ (for voxel sizes $0.5-10 \mu \mathrm{m}$ ). This means that, despite the nondestructive capability, often samples need some form of sectioning to allow a higher resolution to be obtained.

The limitation on minimum detectable pore size was also mentioned in the related section above, where it was mentioned that pores $>3$ voxels wide are the minimum size that can be reliably detected. Hence, the smallest pore size detected in a $10 \mathrm{~mm}$ part, where voxel size is $10 \mu \mathrm{m}$, is $30 \mu \mathrm{m}$ in diameter. These sizes relate to the largest diameter of the pore, unlike $2 \mathrm{D}$ sections that often detect smaller pores, due to the noncentral sectioning of the pore. Comparisons between 3D and 2D analyses should therefore be handled with care and might lead to misinterpretation. 
The obtained image quality is strongly dependent on a proper choice of scan parameters and on scan time, and hence cost. As mentioned previously, this is one of the major drawbacks of microCT. Fast scanning reduces image quality, which can limit the ability to detect features or make suitable conclusions. High scan quality allows more complex analysis methods and reduces the load on image processing. Therefore, an ideal compromise of scan time and image processing time is required, which ideally needs to be developed on an individual basis for each type of application. In the next section, suggestions are made with respect to types of analysis possible at low, medium, and high time investment and hence cost, giving an idea of what is possible for effective microCT analysis of AM parts.

\section{Strategies for Effective Use of microCT}

In this section, the strategies for best use of microCT in AM applications are summarized, considering the current state of the art as reviewed above. These strategies will vary slightly according to the type of microCT equipment used, computing power available, software used for analysis, and quality of the scan data obtained (which can vary with skill and experience of the operator). Nevertheless, it provides a general guide for the AM engineer to understand the microCT technology and realize how best to make use of it. In all cases, it makes sense to share facilities and make use of existing expertise, such as those available in multiuser or service facilities.

\section{Fast options}

For fast and hence low-cost inspections, especially for batches of samples, microCT systems can make 2D X-ray images almost on demand as mentioned above. This allows inspection of many parts per hour, and based on the X-ray inspections, full microCT scans can be done on suspected defective parts.

Similar to above, and more suitable to AM parts with complex designs, fast microCT scans can be performed at reduced quality and resolution to simply view the interior of parts for major flaws. Up to four parts per hour can typically be handled in this way, and only basic image stacks generated for basic inspection (no dimensional analysis). As mentioned previously, higher quality scans can subsequently be done on selected parts in a batch, when required.

Generally, any type of scan, where the data themselves are only viewed for qualitative assessments, is relatively simple and requires only scan time investment, without the need for specialized analysis procedures or interpretation of 3D data. The slice images are viewed in sequence from one side to the other side of the part, which can be typically done in three axes.

\section{Medium time investment}

Due to the required image quality for porosity or inclusion analysis, and the type of layered and small defects found in AM parts, reasonably high image quality is required with scan times $1-2 \mathrm{~h}$ on a whole part, excluding any postprocessing of the data (which can be even more time-consuming). The use of witness specimens overcomes the resolution limit, allowing higher resolution than possible on the whole part, in most cases. If statistical analysis demonstrates that the witness specimen does not contain critical pore sizes, as described in
Refs., ${ }^{60,96}$ then a pass decision can be made on the larger parts of the same build.

This assessment might still be subject to manual inspection of microCT scans of the larger parts (despite the poorer resolution and image quality), as the presence of major flaws and build defects can easily be identified in microCT scans. This is important since extrapolation of porosity information from a witness specimen is not necessarily correct, as additional problems may arise in the build, for example, due to complex build angles, supports, or lack of support—such a disparity between witness specimen and actual part is demonstrated in figure 3 in a study by Seifi et al. ${ }^{97}$ Nevertheless, the use of witness specimens reduces the dependence and heavy time investment on image quality of the whole part and provides the potential to standardize the shape (of witness specimens) and scan settings for such witness specimens, providing potentially a route to much-needed standardization in the field.

Generally, a useful and reasonable time investment is to optimize process parameters of a new AM system or a new AM material parameter set, using small test parts subjected to microCT, to ensure lack of defects in a processing range (power, speed, layer thickness) for a given type of powder. It may also be useful to microCT powders from new suppliers or to do microCT tests of powder samples from every loaded batch of prints, as evidence; and for later possible correlation of build defects with powder quality. One way of reducing the cost for batches is the automation of analysis tasks, using a fixed recipe of steps - this not only saves time but also assists in standardization and comparison of results irrespective of the system or operator used.

\section{Specialized applications and high time investment for critical parts}

For defect analysis of the most critical parts, the best possible scan quality is required. Higher scan times lead to reduced noise and hence better detection of defects. To find optimized parameters for custom parts of varying materials, this can often translate into repeats of scans, when the orientation is such that artifacts are present, or when scan settings need refinement. This process can be streamlined using reference objects (e.g., with intentional cavities) to prove that sufficient image quality is available to detect the defects of interest. It also assists when optimized parameters are developed for a new type of specimen, which requires some method development in scanning and reconstruction parameters and image analysis workflow.

In addition to budgeting for scanning parameter optimization, the analysis workflow often requires custom development for an application, especially to remove noise and ensure no important features are "smoothed over." The best solution is to find the optimized recipe for a particular application and always follow this same recipe for subsequent similar parts. This approach ensures consistency of quality; one example where this was done successfully is as part of the ISO accreditation of the medical implant production by AM at CRPM in the Central University of Technology, South Africa. Each medical implant produced at this facility is subject to a microCT quality inspection according to some fixed criteria of scan settings, to ensure lack of major flaws.

For any kind of dimensional measurement, lack of image artifacts is even more crucial than for porosity analysis. This 
requires high scan quality and optimization of parameters. The same arguments as above hold, but smaller parts and higher quality are required, resulting in at least $1-2 \mathrm{~h}$ of scan time and the same analysis time per part.

For porosity hotspots, structural mechanics simulations based on microCT data, deformation or other 4D CT analysis, most time investment is on the data analysis. This can be implemented using fast scans, but this increases the time investment as denoising is required. For most advanced applications, the best possible scan quality is required. As mentioned above, some workflows can be developed to be followed as a recipe, which can allow cost-effective solutions for this type of analysis.

For surface topography measurements, small sections or small samples need to be scanned, or region of interest scans need to be conducted to allow the field of view and resolution to be appropriate for surface analysis, with at least $35 \mu \mathrm{m}$ voxel size or better, depending on the roughness values expected. Highest possible scan quality is required, and processing the data requires more time investment than the scan itself. This is therefore suggested for small coupon samples only.

For powder analysis, similar to the above arguments, a field of view as small as possible and voxel size as small as possible are required. For the example presented, $1.5 \mu \mathrm{m}$ voxel size allowed the full characterization of the 1-mm-wide sample of powder investigated, but some powder might require higher resolution to allow the same quantitative analysis. For such small voxel sizes, often scan times can become excessively long. Image processing in this case requires denoising and advanced image analysis to virtually separate touching particles.

\section{Summary of microCT capabilities}

Table 1 below summarizes the type of microCT scans and analysis capabilities based on the goal of the work, in broad terms. By keeping to these guidelines, the most cost-effective microCT solution is found.

\section{Development of standards}

The challenges related to developing industry standards for nondestructive testing of AM parts are summarized in Refs. ${ }^{97,98}$ These authors suggest a variety of methods to improve the current lack of standards, including guidelines on how to seed natural flaws into test parts, and thereby demonstrate the ability to detect these flaws, and the use of intentional

Table 1. Type of Microcomputed Tomography Scans and Analysis Capabilities BASED ON THE GOAL OF THE WORK

\begin{tabular}{|c|c|c|c|}
\hline Aim of work & Sample requirement & MicroCT strategy & $\begin{array}{c}\text { Time investment } \\
\text { per part }\end{array}$ \\
\hline Routine quick inspection & $\begin{array}{l}\text { Typically }<200 \mathrm{~mm} \text {, smaller } \\
\text { is better }\end{array}$ & $\begin{array}{l}\text { Fast scans for major flaws only, with } \\
\text { selected parts subjected to high-quality } \\
\text { inspections. }\end{array}$ & Low \\
\hline Quality inspection & $\begin{array}{l}\text { Typically }<100 \mathrm{~mm} \text {, smaller } \\
\text { is better }\end{array}$ & $\begin{array}{l}\text { Scans and analyses according to set } \\
\text { criteria, adhering to quality standards. }\end{array}$ & Low-medium \\
\hline Process optimization & $\begin{array}{l}\text { Ideally small test coupons and } \\
\text { powder samples }\end{array}$ & $\begin{array}{l}\text { High-quality scans for powder quality } \\
\text { inspection (lack of porosity, } \\
\text { irregularity, or impurity), porosity or } \\
\text { build defects (identify build defect type } \\
\text { and relate to process parameters), } \\
\text { surface roughness assessment, } \\
\text { volumetric density (e.g., for alloys). }\end{array}$ & Medium \\
\hline Critical part inspection & $\begin{array}{l}\text { Ideally the part without } \\
\text { support structures after heat } \\
\text { treatment, with associated } \\
\text { witness specimen (rod built } \\
\text { in same direction as critical } \\
\text { part) }\end{array}$ & $\begin{array}{l}\text { Highest possible resolution and quality } \\
\text { scan for part size to identify flaws and } \\
\text { build deviations from CAD design. } \\
\text { Higher resolution scans of witness } \\
\text { specimen to identify possible layer } \\
\text { defects or process defects that may be } \\
\text { present in complex part. Critical areas } \\
\text { of part scanned at higher resolution } \\
\text { using region of interest scans. }\end{array}$ & Medium-high \\
\hline Detailed R\&D & $\begin{array}{l}\text { Smallest part sizes for best } \\
\text { resolution and image } \\
\text { quality }\end{array}$ & $\begin{array}{l}\text { Highest possible scan resolution and } \\
\text { quality, including time investment in } \\
\text { reconstruction and image analysis } \\
\text { optimization. Specific workflows can } \\
\text { be optimized, which can be used as } \\
\text { routine methods for each type of } \\
\text { material and part. } \\
\text { Advanced analyses such as detailed } \\
\text { porosity correlation with wall thickness } \\
\text { and local porosity hotspots, image- } \\
\text { based simulations, and more. }\end{array}$ & High \\
\hline
\end{tabular}


flaws as watermarks in parts, also to act as proof of detection capability. They also suggest further studies of the classification of defect types and causes in AM and further studies of the effect of defects on mechanical properties. These authors also demonstrate that coupon specimens and actual parts may vary in their defect distribution due to build geometry.

Nevertheless, the use of test coupons or witness specimens is a good idea, as higher resolution imaging is possible on these parts compared with larger complex parts, allowing smaller defects to be characterized. This may assist in identifying process parameter errors (lack of fusion or keyhole mode) or build mistakes, such as layer defects (which typically run through the entire build). Some recent studies using witness specimens have identified methods to predict fatigue life of the part based on statistical analysis of pores detected in witness specimens. ${ }^{60,96}$

Currently there are standards being developed for nondestructive testing of AM parts ${ }^{99}$ and specifically for seeding of flaws intentionally into parts for detection proof of concept. ${ }^{100}$ Existing protocols and best practice for industrial microCT are also in place and used widely already. ${ }^{12}$ Considering the current state of the art as reviewed here, we suggest the following to aid in standardization:

- Ten millimeter cubes can be used for high-resolution and quality microCT scans to improve process parameters, identify process defects clearly, quantify extent and type of porosity or inclusions present, assess surface roughness on top and sides of cube, and measure the volumetric density (especially for alloys).

- Witness specimen built in same build as critical part15-mm-diameter cylinder with height corresponding to maximum height of the part.

The cubes can assist in obtaining information for process optimization, the witness specimens can provide information on the specific pores present in the build and which can be in the complex part, including possible layer defects, and the complex part must be analyzed at the highest resolution possible to ensure no additional flaws are detected. In very critical applications, small coupons should be produced with seeded flaws that can be scanned in the same volume as the critical part, as proof of detection capability for small defects.

As the field develops and workflows become standardized, the produced parts will improve in quality and this will contribute to the wider adoption of AM in industry.

\section{Conclusions and Future Perspectives}

It has been demonstrated in this review that microCT can be used for various applications in AM, more than most researchers and engineers in AM realize. It can be used not only for high-quality detailed analysis of single parts but also cost effectively check for major flaws using fast scans with minimal postprocessing. The capabilities extend past individual part inspections to optimization of process parameters using small test coupons, and using witness specimens for indirect inspections of builds. This latter aspect allows for prediction of properties of the complex part, based on the detailed defect analysis of the witness specimen.

Incorporating microCT into a holistic quality control for AM can be done effectively and reasonably simply, despite concerns over the cost of the technique. This extends to mi-
croCT analysis of powder, not only checking for porosity or sphericity but also simply checking for impurities such as denser particles that can lead to inclusions in the built parts. It was demonstrated that scans can be done at varying parameters, for different analysis goals, and can therefore be customized to individual quality inspection workflows.

New methods of using microCT in the field of AM continue to be developed and refined and this adds value and confidence to the use of microCT in the AM field, and adds to the standardization of workflows. Clearly microCT is crucial to the holistic quality analysis and improvement of AM parts and the synergy between these technologies will continue.

\section{Acknowledgments}

This work is based on research supported by the Collaborative Program in Additive Manufacturing and South African Research Chairs Initiative of the Department of Science and Technology and the National Research Foundation of South Africa.

\section{Author Disclosure Statement}

No competing financial interests exist.

\section{References}

1. Gu DD, Meiners W, Wissenbach K, et al. Laser additive manufacturing of metallic components: Materials, processes and mechanisms. Int Mater Rev 2012;57:133-164.

2. DebRoy T, Wei HL, Zuback JS, et al. Additive manufacturing of metallic components-Process, structure and properties. Prog Mater Sci 2018;92:112-224.

3. Yadroitsev I, Gusarov A, Yadroitsava, I, et al. Single track formation in selective laser melting of metal powders. J Mater Process Technol 2010;210:1624-1631.

4. Maire E, Withers PJ. Quantitative X-ray tomography. Int Mater Rev 2014;59:1-43.

5. De Chiffre L, Carmignato S, Kruth JP, et al. Industrial applications of computed tomography. CIRP Ann Manuf Technol 2014;63:655-677.

6. Leary M, Mazur M, Elambasseril J, et al. Selective laser melting (SLM) of AlSi12Mg lattice structures. Mater Des 2016;98:344-357.

7. Valdez M, Kozuch C, Faierson EJ, et al. Induced porosity in Super Alloy 718 through the laser additive manufacturing process: Microstructure and mechanical properties. J Alloys Compd 2017;725:757-764.

8. Thompson A, Maskery I, Leach RK. X-ray computed tomography for additive manufacturing: A review. Meas Sci Technol 2016;27:072001.

9. Kak AC, Slaney M. Principles of Computerized Tomographic Imaging. New York: Society for Industrial and Applied Mathematics, 2001.

10. Schoeman L, Williams $\mathrm{P}$, du Plessis A, et al. X-ray microcomputed tomography $(\mu \mathrm{CT})$ for non-destructive characterisation of food microstructure. Trends Food Sci Technol 2016;47:10-24.

11. du Plessis A, Broeckhoven C, Guelpa A, et al. Laboratory $\mathrm{X}$-ray micro-computed tomography: A user guideline for biological samples. Gigascience 2017;6:1-11.

12. ASTM E1570-11. ASTM E1570-11, Standard Practice for Computed Tomographic (CT) Examination. West Conshohocken, PA: ASTM International, 2011. 
13. du Plessis A, le Roux SG, Guelpa A. The CT Scanner Facility at Stellenbosch University: An open access X-ray computed tomography laboratory. Nucl Instrum Methods Phys Res B 2016;384:42-49.

14. Seifi M, Salem AA, Satko DP, et al. Effects of HIP on microstructural heterogeneity, defect distribution and mechanical properties of additively manufactured EBM Ti48Al-2Cr-2Nb. J Alloys Compd 2017;729:1118-1135.

15. Scarlett NVY. Tyson P, Fraser D, et al. Synchrotron X-ray CT characterization of titanium parts fabricated by additive manufacturing. Part I. Morphology. J Synchrotron Radiat 2016;23:1006-1014.

16. Scarlett NVY. Tyson P, Fraser D, et al. Synchrotron X-ray CT characterization of titanium parts fabricated by additive manufacturing. Part II. Defects. J Synchrotron Radiat 2016;23:1015-1023.

17. Feldkamp LA, Davis LC, Kress JW. Practical cone-beam algorithm. J Opt Soc Am A 1984;1:612.

18. Biguri A, Dosanjh M, Hancock S, et al. TIGRE: A MATLAB-GPU toolbox for CBCT image reconstruction. Biomed Phys Eng Express 2016;2:55010.

19. Khairallah SA, Anderson AT, Rubenchik A, et al. Laser powder-bed fusion additive manufacturing: Physics of complex melt flow and formation mechanisms of pores, spatter, and denudation zones. Acta Mater 2016;108: 36-45.

20. Grasso M, Demir AG, Previtali B, et al. In situ monitoring of selective laser melting of zinc powder via infrared imaging of the process plume. Robot Comput Integr Manuf 2018;49:229-239.

21. Yadroitsau I. Selective Laser Melting: Direct Manufacturing of 3D-Objects by Selective Laser Melting of Metal Powders. Saarbrucken, Germany: LAP LAMBERT Academic Publishing, 2009.

22. Cacace S, Demir AG, Semeraro Q. Densification mechanism for different types of stainless steel powders in selective laser melting. Procedia CIRP 2017;62:475-480.

23. Chen H, Wei Q, Wen S, et al. Flow behavior of powder particles in layering process of selective laser melting: Numerical modeling and experimental verification based on discrete element method. Int J Mach Tools Manuf 2017; 123:146-159.

24. Tan JH, Wong WLE, Dalgarno KW. An overview of powder granulometry on feedstock and part performance in the selective laser melting process. Addit Manuf 2017;18: 228-255.

25. Yadroitsev I, Bertrand P, Smurov I. Parametric analysis of the selective laser melting process. Appl Surf Sci 2007; 253:8064-8069.

26. Spierings AB, Herres N, Levy G. Influence of the particle size distribution on surface quality and mechanical properties in AM steel parts. Rapid Prototyp J 2011;17:195202.

27. Yadroitsev I, Yadroitsava I, Bertrand $\mathrm{P}$, et al. Factor analysis of selective laser melting process parameters and geometrical characteristics of synthesized single tracks. Rapid Prototyp J 2012;18:201-208.

28. Matilainen VP, Piili H, Nyrhilä O. Preliminary Investigation of Keyhole Phenomena during single layer fabrication in laser additive manufacturing of stainless steel. Phys Procedia 2015;78:377-387.

29. Buchbinder D, Schleifenbaum H, Heidrich S, et al. High power selective laser melting (HP SLM) of aluminum parts. Phys Procedia 2011;12:271-278.
30. Kasperovich G, Haubrich J, Gussone J, et al. Correlation between porosity and processing parameters in TiAl6V4 produced by selective laser melting. Mater Des 2016;105: 160-170.

31. King WE, Barth HD, Castillo VM, et al. Observation of keyhole-mode laser melting in laser powder-bed fusion additive manufacturing. J Mater Process Technol 2014; 214:2915-2925.

32. Aboulkhair NT, Everitt NM, Ashcroft I, et al. Reducing porosity in AlSi10Mg parts processed by selective laser melting. Addit Manuf 2014;1-4:77-86.

33. Panwisawas C, Perumal B, Ward RM, et al. Keyhole formation and thermal fluid flow-induced porosity during laser fusion welding in titanium alloys: Experimental and modelling. Acta Mater 2017;126:251-263.

34. Cunningham R, Narra SP, Montgomery C, et al. Synchrotronbased X-ray microtomography characterization of the effect of processing variables on porosity formation in laser powerbed additive manufacturing of Ti-6Al-4V. JOM 2017;69: 479-484.

35. Slotwinski JA, Garboczi EJ, Hebenstreit KM. Porosity measurements and analysis for metal additive manufacturing process control. J Res Natl Inst Stand Technol 2014; 119:494-528.

36. Tang M, Pistorius PC, Beuth JL. Prediction of lack-offusion porosity for powder bed fusion. Addit Manuf 2017; 14:39-48.

37. Yadroitsev I, Smurov I. Surface morphology in selective laser melting of metal powders. Phys Procedia 2011;12: 264-270.

38. Ziółkowski G, Chlebus E, Szymczyk P, et al. Application of X-ray CT method for discontinuity and porosity detection in $316 \mathrm{~L}$ stainless steel parts produced with SLM technology. Arch Civ Mech Eng 2014;14:608-614.

39. Rashid R, Masood SH, Ruan D, et al. Effect of scan strategy on density and metallurgical properties of 17-4PH parts printed by selective laser melting (SLM). J Mater Process Technol 2017;249:502-511.

40. Shi Q, Gu D, Xia M, et al. Effects of laser processing parameters on thermal behavior and melting/solidification mechanism during selective laser melting of TiC/Inconel 718 composites. Opt Laser Technol 2016;84:9-22.

41. Ponnusamy P, Masood SH, Ruan D, et al. Statistical analysis of porosity of 17-4PH alloy processed by selective laser melting. IOP Conf Ser Mater Sci Eng 2017;220:12001.

42. Kim FH, Moylan SP, Garboczi EJ, et al. Investigation of pore structure in cobalt chrome additively manufactured parts using X-ray computed tomography and three-dimensional image analysis. Addit Manuf 2017; $17: 23-38$

43. Panwisawas C, Qiu CL, Sovani Y, et al. On the role of thermal fluid dynamics into the evolution of porosity during selective laser melting. Scripta Mater 2015;105:14-17.

44. Cai X, Malcolm AA, Wong BS, et al. Measurement and characterization of porosity in aluminium selective laser melting parts using X-ray CT. Virtual Phys Prototyp 2015; 10:195-206.

45. Cunningham R, Nicolas A, Madsen J, et al. Analyzing the effects of powder and post-processing on porosity and properties of electron beam melted Ti-6Al-4V. Mater Res Lett 2017;5:516-525.

46. Tillmann W, Schaak C, Nellesen J, et al. Hot isostatic pressing of IN718 components manufactured by selective laser melting. Addit Manuf 2017;13:93-102. 
47. Zhang B, Liao H, Coddet C. Effects of processing parameters on properties of selective laser melting $\mathrm{Mg}-$ 9\%Al powder mixture. Mater Des 2012;34:753-758.

48. Kouprianoff D, Luwes N, Newby E, et al. On-line monitoring of laser powder bed fusion by acoustic emission: Acoustic emission for inspection of single tracks under different powder layer thickness. In: 2017 Pattern Recognition Association of South Africa and Robotics and Mechatronics (PRASA-RobMech). New York, IEEE, 2017; pp. 203-207.

49. Karme A, Kallonen A, Matilainen VP, et al. Possibilities of CT scanning as analysis method in laser additive manufacturing. Phys Procedia 2015;78:347-356.

50. Damon J, Dietrich S, Vollert F, et al. Process dependent porosity and the influence of shot peening on porosity morphology regarding selective laser melted AlSi10Mg parts. Addit Manuf 2018;20:77-89.

51. Wits WW, Carmignato S, Zanini F, et al. Porosity testing methods for the quality assessment of selective laser melted parts. CIRP Ann 2016;65:201-204.

52. du Plessis A, Le Roux SG, Els J, et al. Application of microCT to the non-destructive testing of an additive manufactured titanium component. Case Stud Nondestruct Test Eval 2015;4:1-7.

53. du Plessis A, Rossouw P. Investigation of porosity changes in cast Ti6Al4V rods after hot isostatic pressing. J Mater Eng Perform 2015;24:3137-3141.

54. Konečná R, Kunz L, Nicoletto G, et al., A. Long fatigue crack growth in Inconel 718 produced by selective laser melting. Int J Fatigue 2016;92:499-506.

55. Yadroitsev I, Krakhmalev P, Yadroitsava I, et al. Qualification of Ti6Al4V ELI alloy produced by laser powder bed fusion for biomedical applications. JOM 2018;70:372-377.

56. Tammas-Williams S, Zhao $\mathrm{H}$, Léonard $\mathrm{F}$, et al. XCT analysis of the influence of melt strategies on defect population in Ti-6Al-4V components manufactured by Selective Electron Beam Melting. Mater Charact 2015;102:47-61.

57. Maskery I, Aboulkhair K, Corfield MR, et al. Quantification and characterisation of porosity in selectively laser melted Al-Si10-Mg using X-ray computed tomography. Mater Charact 2016;111:193-204.

58. du Plessis A, Le Roux SG, Booysen G, et al. Quality control of a laser additive manufactured medical implant by X-ray tomography. 3D Print Addit Manuf 2016;3:175-182.

59. du Plessis A, Yadroitsava I, Le Roux SG, et al. Prediction of mechanical performance of Ti6Al4V cast alloy based on microCT-based load simulation. J Alloys Compd 2017; 724:267-274.

60. Romano S, Brandão A, Gumpinger J, et al. Qualification of AM parts: Extreme value statistics applied to tomographic measurements. Mater Des 2017;131:32-48.

61. du Plessis A, Le Roux SG, Booysen G, et al. Directionality of cavities and porosity formation in powder-bed laser additive manufacturing of metal components investigated using X-ray tomography. 3D Print Addit Manuf 2016;3:48-55.

62. Kouprianoff D, du Plessis A, Yadroitsava I, et al. Nondestructive testing of the parts manufactured by direct metal laser sintering. Central University of Technology, Free State DSpace Repository. 2016. Available at: http:// hdl.handle.net/11462/1213 Accessed July 13, 2018.

63. Siddique S, Awd M, Tenkamp J, et al. Development of a stochastic approach for fatigue life prediction of AlSi12 alloy processed by selective laser melting. Eng Fail Anal 2017;79:34-50.
64. Siddique S, Imran M, Rauer $\mathrm{M}$, et al. Computed tomography for characterization of fatigue performance of selective laser melted parts. Mater Des 2015;83:661669.

65. Benedetti M, Fontanari V, Bandini M, et al. Low- and high-cycle fatigue resistance of Ti-6Al-4V ELI additively manufactured via selective laser melting: Mean stress and defect sensitivity. Int J Fatigue 2018;107:96-109.

66. Domfang Ngnekou JN, Nadot Y, Henaff G, et al. Influence of defect size on the fatigue resistance of AlSi10Mg alloy elaborated by selective laser melting (SLM). Procedia Struct Integr 2017;7:75-83.

67. Naragani D, Sangid MD, Shade PA, et al. Investigation of fatigue crack initiation from a non-metallic inclusion via high energy x-ray diffraction microscopy. Acta Mater 2017; 137:71-84.

68. Lou X, Andresen PL, Rebak RB. Oxide inclusions in laser additive manufactured stainless steel and their effects on impact toughness and stress corrosion cracking behavior. J Nucl Mater 2018;499:182-190.

69. du Plessis A, Meincken M, Seifert T. Quantitative determination of density and mass of polymeric materials using microfocus computed tomography. J Nondestruct Eval 2013;32:413-417.

70. Villarraga-Gómez H, Lee C, Smith ST. Dimensional metrology with X-ray CT: A comparison with CMM measurements on internal features and compliant structures. Precis Eng 2018;51:291-307.

71. Ferrucci M, Leach RK, Giusca C, et al. Towards geometrical calibration of x-ray computed tomography systems-A review. Meas Sci Technol 2015;26:92003.

72. Snyder JC, Stimpson CK, Thole KA, et al. Build direction effects on microchannel tolerance and surface roughness. J Mech Des 2015;137:111411.

73. Brackett D, Ashcroft I, Hague R. Topology optimization for additive manufacturing. In: 22nd Annual International Solid Freeform Fabrication Symposium-An Additive Manufacturing Conference, Austin, TX, SFF 2011. pp. 348-362.

74. Hildebrand T, Ruegsegger P. A new method for the modelindependent assessment of thickness in three-dimensional images. J Microsc 1997; 185:67-75.

75. Thomsen JS, Laib A, Koller B, et al. Stereological measures of trabecular bone structure: Comparison of 3D micro computed tomography with 2D histological sections in human proximal tibial bone biopsies. J Microsc 2005; 218:171-179.

76. Krakhmalev P, Fredriksson G, Yadtroitsava I, et al. Deformation behavior and microstructure of Ti6Al4V manufactured by SLM. Phys Procedia 2016;83:778788.

77. Bay BK, Smith TS, Fyhrie DP, et al. Digital volume correlation: Three-dimensional strain mapping using Xray tomography. Exp Mech 1999;39:217-226.

78. du Plessis A, Broeckhoven C, Le Roux SG. Snake fangs: 3D morphological and mechanical analysis by microCT, simulation, and physical compression testing. Gigascience 2018;7:1-8.

79. Buffiere JY, Maire E, Adrien J, et al. In situ experiments with $\mathrm{x}$ ray tomography: An attractive tool for experimental mechanics. Exp Mech 2010;50:289-305.

80. Tammas-Williams S, Withers PJ, Todd I, et al. Porosity regrowth during heat treatment of hot isostatically pressed additively manufactured titanium components. Scripta Mater 2016;122:72-76. 
81. Triantaphyllou A, Giusca CL, Macaulay GD, et al. Surface texture measurement for additive manufacturing. Surf Topogr 2015;3:24002.

82. Townsend A, Senin N, Blunt L, et al. Surface texture metrology for metal additive manufacturing: A review. Precis Eng 2016;46:34-47.

83. Kerckhofs G, Pyka G, Moesen M, et al. High-resolution microfocus X-ray computed tomography for 3D surface roughness measurements of additive manufactured porous materials. Adv Eng Mater 2013;15:153-158.

84. Pyka G, Kerckhofs G, Papantoniou I, et al. Surface roughness and morphology customization of additive manufactured open porous Ti6Al4V structures. Materials (Basel) 2013;6:4737-4757.

85. Thompson A, Senin N, Giusca C, et al. Topography of selectively laser melted surfaces: A comparison of different measurement methods. CIRP Ann 2017;66:543-546.

86. Thompson A, Senin N, Maskery I, et al. Internal surface measurement of metal powder bed fusion parts. Addit Manuf 2018;20:126-133.

87. Tammas-Williams S, Withers PJ, Todd I, et al. The influence of porosity on fatigue crack initiation in additively manufactured titanium components. Sci Rep 2017;7:7308.

88. Fieres J, Schuman P, Reinhart C. Predicting failure in additively manufactured parts using X-ray computed tomography and simulation. Procedia Eng 2018;213:69-78.

89. du Plessis A, Yadroitsev I, Yadroitsava I, et al. S. Analysis of diagonal and rhombic Dmls Ti6al4v (Eli) lightweight structures. Opt Laser Technol 2018; In press.

90. du Plessis A, Olawuyi BJ, Boshoff WP, et al. Simple and fast porosity analysis of concrete using X-ray computed tomography. Mater Struct Constr 2016;49:553-562.

91. Guelpa A, du Plessis A, Manley M. A high-throughput Xray micro-computed tomography $(\mu \mathrm{CT})$ approach for measuring single kernel maize (Zea mays L.) volumes and densities. J Cereal Sci 2016;69:321-328.

92. Heim K, Bernier F, Pelletier R, et al. High resolution pore size analysis in metallic powders by X-ray tomography. Case Stud Nondestruct Test Eval 2016;6:45-52.
93. Bernier F, Tahara R, Gendron M. Additive manufacturing powder feedstock characterization using X-ray tomography. Met Powder Rep 2018;73:158-162.

94. Rozendaal A, le Roux SG, du Plessis A, et al. Grade and product quality control by microCT scanning of the world class Namakwa Sands Ti-Zr placer deposit West Coast, South Africa: An orientation study. Miner Eng 2018;116: 152-162.

95. Dzogbewu TC, Yadroitsev I, Krakhmalev P, et al. Optimal process parameters for in situ alloyed ti15mo structures by laser powder bed fusion. In: Annual International Solid Freeform Fabrication Symposium. Austin, TX, 2017.

96. Romano S, Brückner-Foit A, Brandão A, et al. Fatigue properties of AlSi10Mg obtained by additive manufacturing: Defect-based modelling and prediction of fatigue strength. Eng Fract Mech 2017;187:165-189.

97. Seifi M, Gorelik M, Waller J, et al. Progress towards metal additive manufacturing standardization to support qualification and certification. JOM 2017;69:439-455.

98. Seifi M, Salem A, Beuth J, et al. Overview of materials qualification needs for metal additive manufacturing. JOM 2016;68:747-764.

99. ASTM WK47031. Standard Guide for Post-Process Nondestructive Testing of Metal Additively Manufactured Parts Used in Aerospace Applications. West Conshohocken, PA: ASTM International, 2018.

100. ASTM WK56649. Standard Practice/Guide for Intentionally Seeding Flaws in Additively Manufactured (AM) Parts. West Conshohocken, PA: ASTM International, 2018.

Address correspondence to: Anton du Plessis CT Scanner Facility Stellenbosch University PO Sauer Stellenbosch 7602 South Africa

E-mail: anton2@sun.ac.za 